Cahiers $d u$ MONDE RUSSE

\section{Cahiers du monde russe}

Russie - Empire russe - Union soviétique et États indépendants

\title{
Administration, politique et techniques
}

Réflexions sur la matérialité des pratiques administratives dans la

Russie stalinienne (1922-1940)

Yves Cohen

\section{(2) OpenEdition}

\section{Journals}

Édition électronique

URL : https://journals.openedition.org/monderusse/8610

DOI : $10.4000 /$ monderusse. 8610

ISSN : $1777-5388$

Éditeur

Éditions de l'EHESS

\section{Édition imprimée}

Date de publication : 1 avril 2003

Pagination : 269-308

ISBN : 2-7132-1813-6

ISSN : $1252-6576$

Référence électronique

Yves Cohen, "Administration, politique et techniques », Cahiers du monde russe [En ligne], 44/2-3 |

2003, mis en ligne le 01 janvier 2007, consulté le 02 septembre 2022. URL : http://

journals.openedition.org/monderusse/8610; DOI : https://doi.org/10.4000/monderusse.8610 


\section{CAIR N}

chercher : repérer : avancer

Cet article est disponible en ligne à l'adresse :

http://www.cairn.info/article.php?ID REVUE=CMR\&ID NUMPUBLIE=CMR 442\&ID ARTICLE=CMR 4420269

Administration, politique et techniques. Réflexions sur la matérialité des pratiques administratives dans la Russie stalinienne (1922-1940)

par Yves COHEN

\section{| Editions de l'EHESS | Cahiers du monde russe}

\section{3/2-3 Vol 44}

ISSN 1252-6576 | ISBN 2713218136 | pages 269 à 308

Pour citer cet article :

- COHEN Y., Administration, politique et techniques. Réflexions sur la matérialité des pratiques administratives dans la Russie stalinienne (1922-1940), Cahiers du monde russe 2003/2, Vol 44, p. 269-308.

Distribution électronique Cairn pour les Editions de l'EHESS.

(C) Editions de l'EHESS. Tous droits réservés pour tous pays.

La reproduction ou représentation de cet article, notamment par photocopie, n'est autorisée que dans les limites des conditions générales d'utilisation du site ou, le cas échéant, des conditions générales de la licence souscrite par votre établissement. Toute autre reproduction ou représentation, en tout ou partie, sous quelque forme et de quelque manière que ce soit, est interdite sauf accord préalable et écrit de l'éditeur, en dehors des cas prévus par la législation en vigueur en France. Il est précisé que son stockage dans une base de données est également interdit. 
YVES COHEN

\title{
ADMINISTRATION, POLITIQUE ET TECHNIQUES
}

\author{
Réflexions sur la matérialité des pratiques administratives \\ dans la Russie stalinienne (1922-1940)
}

\begin{abstract}
Administration, politique et police
Si l'histoire de l'administration importe pour l'histoire de l'Union soviétique, ce n'est pas simplement du fait que l'administration est massivement là et qu'elle se présente à l'enquête historique ${ }^{1}$. L'histoire de l'URSS offre des caractéristiques particulières qui imposent de s'intéresser de façon toute spéciale à l'administration. Cette nécessité ne tient pas non plus au fait que l'Union soviétique expose au plus haut point ce phénomène du $\mathrm{XX}^{\mathrm{e}}$ siècle qu'est la croissance de la bureaucratie dans l'État comme dans l'économie. Il ne s'agit pas d'illustrer avec l'exemple soviétique une histoire wébérienne de la rationalité et de l'irrationalité bureaucratiques. Si l'histoire de l'administration importe dans l'histoire de l'Union soviétique, c'est que, comme Moshe Lewin le rappelle judicieusement dans ce volume et dans son dernier livre, toute politique y est étouffée ${ }^{2}$. En d'autres termes, il n'y reste qu'à administrer et l'administration y consiste à étouffer activement la politique. Le gouvernement bolchevik a en effet disqualifié toute politique dans un sens précis, celui où la politique est la mise au jour de la mésentente (selon l'heureuse formulation de Jacques Rancière). Le pouvoir bolchevik ne laisse d'emblée aucune place à

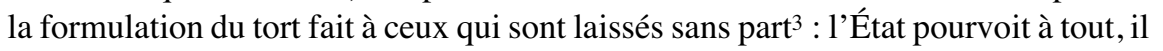

1. Je remercie Alexis Berelowitch, Dorena Caroli, Olivia Gomolinski, Kapil Raj, Brigitte Studer et Guy Thuillier pour leurs précieuses indications.

2. Moshe Lewin, Le siècle soviétique, Paris, Fayard et Le Monde diplomatique, 2003, 526 p.

3. Nicolas Werth, «Un État contre son peuple. Violences, répressions, terreurs en Union soviétique », in Stéphane Courtois, dir., Le livre noir du communisme, Paris, Robert Laffont, 1997, p. 42-295. 
est la voix du prolétariat et du peuple. Le parti est le lieu de la vérité et du droit et ceux-ci n'ont plus place ailleurs que dans la subordination. La seule politique qui subsiste est celle, le plus souvent imaginaire, des classes dirigeantes abattues, de leurs derniers agents et de ceux des pays capitalistes extérieurs. Tout désaccord est réinterprété comme l'expression d'une telle politique. Dès lors, tout l'espace est occupé par la «police », au sens très général de Foucault repris par Rancière, c'està-dire très proche de l'«administration » et aussi, avec quelques nuances sans doute, de la «gestion ». Pour Rancière, la police est « l'ensemble des processus par lesquels s'opèrent l'agrégation et le consentement des collectivités, l'organisation des pouvoirs, la distribution des places et fonctions et les systèmes de légitimation de cette distribution ». Elle est encore « un ordre des corps qui définit les partages entre les modes du faire, les modes d'être et les modes du dire, qui fait que tels corps sont assignés par leur nom à telle place et à telle tâche ; c'est un ordre du visible et du dicible qui fait que telle activité est visible et que telle autre ne l'est pas, que telle parole est entendue comme du discours et telle autre comme du bruit $»^{4}$. La police généralisée dans l'ordre soviétique ne laisse place à aucune parole d'égalité formulée dans d'autres termes que ceux du pouvoir: tous ont formellement la parole, tout prolétaire, tout travailleur, tout élément du peuple, et même tout peuple, car tous parlent par le parti. La politique n'a pas lieu.

Cette élision de la politique est difficile à penser, puisque l'Union soviétique est sans cesse dirigée par le parti communiste, que le communisme est considéré comme l'incarnation même de la politique au $\mathrm{XX}^{\mathrm{e}}$ siècle, que son idéologie veut que toute activité soit acceptée comme politique et donc que le gouvernement par le communisme doit être politique. Or, en premier lieu, le communisme en Union soviétique n'est que le défenseur de hiérarchies constituées et des intérêts particuliers qu'elles portent : cette défense est œuvre de police, au sens large indiqué qui comporte aussi le sens étroit de police politique, œuvre, en d'autres termes, $\mathrm{d}^{\prime}$ ' administration $»^{5}$. Le rôle du parti communiste dans son rapport à l'appareil gouvernemental consiste à verrouiller sans cesse l'irruption de la parole politique, en particulier par une production idéologique ajustée. En second lieu, l'action de l'Internationale communiste puis de la direction mondiale du communisme par le parti soviétique n'apparaît pas tout de suite aux yeux de tous pour ce qu'elle devient très vite - un outil de gestion du salut soviétique - mais comme la porteuse de la mésentente, c'est-à-dire comme l'initiatrice de la politique, ce qu'elle est en fait partiellement, mais ailleurs qu'en URSS : la politique de l'Union soviétique consiste à maintenir et à attiser la politique à l'extérieur (dans les limites qui lui conviennent, assurément) tout en l'étouffant chez soi. Incidemment, ceci fait de l'histoire «administrative » soviétique tout autre chose qu'une histoire non politique, dépolitisée.

4. Jacques Rancière, La Mésentente. Politique et philosophie, Paris, Galilée, 1995, p. 51 et 52.

5. Michel Foucault rappelle dans " "Omnes et singulatim": vers une critique de la raison politique » (1981) que la science de l'administration est enseignée aux XVII' et XVIII' siècle en Allemagne sous le titre de Polizeiwissenschaft (Dits et écrits, vol. 4, Paris, Gallimard, 1994. p. 156). Voir aussi id., « La technologie politique des individus » (1982), ibid., p. 813-828. 
La disqualification de la politique ne laisse plus que de l'administration. L'histoire de l'administration se rapporte dès lors non pas seulement de façon étroite à l'appareil gouvernemental, ni même de façon un peu plus large à toute organisation (puisque les organisations de tous ordres, y compris les partis, en capitalisme comme en socialisme, comportent de l'administration), mais à l'ensemble parti-État et à toutes les sphères d'action qui le composent ${ }^{6}$.

\section{Pratiques administratives $=$ histoire totalitarienne $?$}

L'expression de «pratiques administratives » proposée par ce colloque permet de ne pas faire de l'histoire de l'administration seulement une histoire de structures, de formes, d'organismes et de financements ni non plus seulement une histoire sociale (souvent quantitative et démographique) des différents groupes sociaux qui se constituent dans le cours administratif et qui entrent en conflit pour le pouvoir (origines, formation, revenus, influence...). Par l'étude des pratiques, l'histoire de l'administration peut montrer comment le pouvoir soviétique s'emploie constamment, et avec quel succès, à verrouiller toute irruption de la politique, activité sans cesse renouvelée. C'est en ceci que consiste sa propre politique : en l'écrasement de toute politique. J'admettrai ici ce second sens de politique. Il sera très précisément attribué aux interventions du centre en tant que celui-ci s'emploie sans cesse au renforcement administratif et policier pour mieux juguler la politique au sens d'activation de la mésentente. D'ailleurs, le plus souvent, lorsqu'on rencontre le terme de «politique » dans l'historiographie soviétique, c'est justement à cette remise en ordre venue du centre qu'il renvoie. L'histoire de l'administration peut de la sorte être une histoire politique de l'administration : la politique du pouvoir soviétique consiste à être exclusivement administratif, à absorber tout dans la sphère des pratiques administratives, y compris l'art et le droit, à tout régler par des mesures administratives. Les divergences internes, dites de "politique», se déploient sur fond de l'accord fondamental du déni de la politique (nul espace n'est accordé à la parole libre des «classes sociales » au nom desquelles le parti gouverne et pense, ni non plus à celle des autres, évidemment).

Une histoire des pratiques administratives pourrait cependant faire craindre dès lors de retomber dans une histoire totalitarienne. Et en effet, l'Union soviétique se constitue au fil des années 1920 comme un espace continu de prescription. Paraissant s'appuyer là-dessus, il existe toute une tradition d'étude de l'URSS considérée comme une entreprise, très proche de la littérature qui n'analyse que la dimension de commandement de la société soviétique ${ }^{7}$. Dans une société capitaliste libérale comme la France du Xxe siècle, des sphères de

6. Voir Alessandro Stanziani, L'économie en révolution : le cas russe, 1870-1930, Paris, Albin Michel, 1998, en particulier p. 405.

7. Voir le point récent fait sur cette question dans Cédric Durand, Une analyse institutionnaliste de la coordination industrielle. Étude des transformations de la métallurgie russe (1992-2001), thèse d'économie (sous la direction de Jacques Sapir), Paris, EHESS, 2002, 537 p. 
souveraineté distinctes se découpent: celle de l'État, celle des entreprises privées, celle des associations (même si les frontières sont parfois poreuses). La démocratie politique maintient un certain jeu où la parole d'égalité et la mésentente sur les comptes de justice peuvent trouver à cheminer. En Union soviétique, la société se fait coextensive à l'espace de prescription administrative et cet espace est continu. Il n'est pourtant pas sans reste, comme Alain Blum le montrait naguère pour la famille ${ }^{8}$. Toutefois l'insistance sur l'espace de prescription continu oriente la réflexion sur la continuité et sur l'homologie entre les espaces sociaux administrés plutôt que sur l'effectivité de la prescription. Cette réflexion est pragmatique et attire aussi l'attention sur les pratiques qui constituent ces espaces. Ce sont des pratiques de pouvoir portant l'effort de déjouer sans cesse l'acte politique. David Shearer montre par exemple comment les réformes de la police dans les années 1930 visaient en grande partie à priver le désordre social croissant de toute issue politique ${ }^{9}$. Ce sont aussi les pratiques de tous les acteurs du social qui n'agissent qu'en aménageant la prescription et en ouvrant dès lors bien des espaces collectifs, comme l'historiographie de l'industrie, par exemple, le montre dès les années $1950^{10}$. Dire qu'il y a prescription n'est en rien dire que celle-ci «fonctionne». C'est ouvrir la recherche pour en saisir la source, la logique, les effets et ce contre quoi elle se définit et prolifère. C'est chercher à accéder aux univers pratiques dont relève le jeu de la prescription administrative et à dessiner les conflits qui opposent ces univers différents, loin d'une histoire dont seule la volonté de contrôle total ou de police généralisée donnerait le secret ${ }^{11}$. Il n'en reste pas moins que l'histoire de cette volonté est à faire aussi... comme une histoire de pratiques.

\section{Un idéal télégraphique de gouvernement}

Or le risque d'une interprétation totalitarienne de l'histoire soviétique ne provient pas seulement de l'épaisse historiographie qui l'a promue durant la guerre froide. Il vient de l'histoire même. Moshe Lewin rappelle encore ici que le parti a très tôt été défini par son premier secrétaire général, Stalin, comme une organisation de cadres soumis. Au même XII congrès d'avril 1923 qu'il évoque, Stalin formule de surcroît une sorte d'idéal télégraphique de gouvernement par lequel le parti n'aurait qu'à

8. A. Blum, Naître, vivre et mourir en URSS, 1917-1991, Paris, Plon, 1994, 273 p.

9. David R. Shearer, «Crime and social disorder in Stalin's Russia. A reassessment of the Great Retreat and the origins of mass repression », Cahiers du Monde russe, 39, 1-2, 1998, p. 119-148 (dans « Social disorder, mass repression, and the NKVD during the 1930s », Cahiers du Monde russe, 42, 2-4, 2001, p. 505-534, D. Shearer indique toutefois que ces opérations n'avaient pas eu dans le déclenchement de la Grande Terreur l'importance qu'il lui attribue dans le premier article).

10. Joseph S. Berliner, Factory and manager in the USSR, Cambridge (Mass.), Harvard University Press, 1957, $386 \mathrm{p}$.

11. Voir la tentative d'Alain Blum et Martine Mespoulet, $L$ 'anarchie bureaucratique. Statistique et pouvoir sous Staline, Paris, La Découverte, 2003, 372 p. 
décider en ses instances les plus élevées et communiquer ses instructions au reste de la société. Suivons son propos. Il écrit certes :

[Je] parle du Parti comme d'une avant-garde, et de la classe ouvrière comme de l'armée de notre Parti. On pourrait penser, par analogie, que les rapports sont ici les mêmes qu'à l'armée, c'est-à-dire que le Parti donne des ordres, que ses consignes sont transmises par le télégraphe et que l'armée, c'est-à-dire la classe ouvrière, exécute ces ordres. Une telle façon de voir est foncièrement erronée. Dans le domaine politique, la question est beaucoup plus compliquée.

En quoi la question est-elle plus compliquée que dans la pure transmission militaire des ordres? Ici, Stalin ne formule pas la réserve habituelle de Lenin qui ne manquait pas de rappeler, au moins en 1918, qu'il «faut apprendre à conjuguer l'esprit démocratique des masses laborieuses, tel qu'il se manifeste dans les meetings, impétueux, débordant, pareil à une crue printanière, avec une discipline de fer pendant le travail, avec la soumission absolue pendant le travail à la volonté d'un seul, du dirigeant soviétique », séparant la liberté politique de la totale subordination au travail ${ }^{12}$. Il ne s'agit donc même pas de simplement tenir compte d'une classe ouvrière d'où proviendrait la parole politique : elle n'a plus d'espace ménagé, même dans le seul discours. Le problème réside dans le fait que le parti hérite d'une masse qu'il n'a pas constituée ni entretenue lui-même, contrairement à la sphère militaire où l'armée est construite, formée et nourrie par ceux-là même qui la commandent. Stalin poursuit :

En effet, dans le domaine militaire, c'est le commandement lui-même qui crée l'armée, qui la forme, alors qu'ici, dans le domaine politique, le Parti ne crée pas son armée mais la trouve toute constituée : c'est la classe ouvrière. Il existe encore une autre différence : dans le domaine militaire, le commandement ne se contente pas de créer l'armée, il la nourrit, l'habille et la chausse [...]. Le Parti ne nourrit, ni n'habille, ni ne chausse son armée : la classe ouvrière. C'est pourquoi, en politique, la question est beaucoup plus complexe. Et ce n'est pas la classe ouvrière qui dépend du parti, mais l'inverse.

L'idéal télégraphique n'en est pas moins valide dans ces conditions simplement plus difficiles. Toutefois, au lieu d'évoquer (non métaphoriquement) le télégraphe, Stalin emploie ici des métaphores techniques (celles du réseau d'appareils, de la courroie de transmission et des antennes, cette dernière étant en même temps une métaphore biologique) pour signifier la même chose, le gouvernement par la transmission :

Aussi, dans le domaine politique, l'avant-garde de la classe, c'est-à-dire le Parti, doit-il, pour assumer son rôle dirigeant, s'entourer d'un large réseau [set'] d'appareils de masse sans parti, qui lui servent en quelque sorte d'antennes et

12. V. I. Lénine, «Les tâches immédiates du pouvoir des Soviets », in Problèmes d'organisation de l'économie socialiste, Moscou, Éditions du Progrès, s.d., p. 144 (« Očerednye zadači sovetskoj vlasti », Sočinenja, t. 36, p. 203). 
par l'intermédiaire desquels il transmet sa volonté à la classe ouvrière, de telle sorte que celle-ci, d'une masse éparpillée, devient l'armée du Parti13.

Voici le modèle télégraphique constitué. Pour assurer le gouvernement de la classe ouvrière, il faut ainsi construire des appareils infiniment plus complexes que ne l'est l'armée qui a l'avantage de fabriquer elle-même son corps d'obéissance. Stalin parle de transmission télégraphique des ordres au sens propre pour l'armée et par des réseaux d'appareils avec antennes et courroies de transmission pour la classe ouvrière. Comme il prétend que l'un n'a rien à voir avec l'autre, que l'armée n'est pas la classe ouvrière, on a le sentiment à la première lecture que l'idéal télégraphique ne se rapporte qu'à l'armée et qu'avec la classe ouvrière la relation est politique (ce qu'il ne dit à aucun moment). On est pourtant dans le même rapport de transmission pour ce qui est de la relation entre le parti et la classe ouvrière, et donc dans le même idéal télégraphique ${ }^{14}$. Le parti « dépend » de la classe ouvrière non pas parce que, dans le discours manifeste, Stalin lui reconnaît une parole propre, mais parce que le parti la trouve telle qu'elle est et doit la transformer en une masse de subordination comme les chefs militaires le font de l'armée.

\section{Les pratiques dans leur matérialité}

L'idéal télégraphique de gouvernement de Stalin est une parmi toutes les preuves de l'intention totalitaire du chef bolchevik. Pourtant, comme tout discoursprogramme, celui de Stalin ne dit que ce qu'il dit. Il est une part d'une pratique discursive qui a ses règles et ne dit rien sur ce qui se passe effectivement, en situation ${ }^{15}$. Il est à comprendre dans son occurrence, à sa date et en son lieu. Mais plus encore, dans la mesure où diverses formes du social (militaire et politique) sont ici indissociables du télégraphe ou de mécanismes de transmission, ce discours renforce le besoin de prendre au sérieux à la fois sa dimension technique et pratique et sa relation à une histoire des pratiques d'administration dans cette dimension matérielle même.

Le discours de Stalin invite à considérer l'importance de la matérialité tout à la fois dans les pratiques administratives de toutes sortes, dans les pratiques discur-

13. I. V. Staline, «Rapport d'organisation du Comité central du PC(b)R (17 avril)», in Euvres, Paris, Éditions sociales, 1955, t. 5, p. 173-174 (Sočinenja, t. 5, 1947, p. 198-199).

14. Lenin prône de son côté une fois, pour l'État et « toute » l'économie nationale, l'idéal de la poste, « modèle d'entreprise socialiste [...] actuellement organisée sur le modèle du monopole capitaliste d'État : [...] le mécanisme de gestion sociale y est déjà tout prêt », modèle tout à la fois « technique », d'obéissance et de gestion ( «L'État et la révolution » (1918), Euvres, t. 25, 1957, p. 460-462). Il évoque ailleurs aussi bien la « direction délicate » du chef d'orchestre que la «régularité du mécanisme d'horloge» («Les tâches immédiates...», art. cit., p. 140 ; "Pervonačal'nyj variant stat'i "Očerednye zadači sovetskoj vlasti" » (Première version de l'article "Les tâches immédiates..."), Sočinenja, t. 36, p. 155-158).

15. Stalin évoque aussi la métaphore de l'horloge utilisée avec insistance par Lenin lorsqu'il parle du fonctionnement des entreprises et de la nécessaire subordination absolue au dirigeant soviétique. 
sives qui tentent de mettre le bon ordre dans la pensée du réel et aussi dans la relation entre les unes et les autres. On aurait pu choisir d'autres textes aux propositions voisines, mais celui-ci ne vient pas de n'importe qui et son expression est, de plus, articulée avec une forte pertinence sur un moment décisif de la transformation de la politique en administration (ou en police) et sur l'outillage mental non trivial qui sert à penser ce mouvement.

Un large courant des sciences sociales contemporaines s'attache justement depuis une vingtaine d'années à montrer la part active des objets dans la constitution du social, au point que les objets pourraient en être considérés comme des acteurs à part entière, dotés de leur propre agency : « Les distinctions entre conversations, textes, techniques, corps sont essentielles. Mais il n'existe aucune raison a priori pour en exclure certains de la participation à la dynamique du collectif : tous ces éléments, tous ces matériaux contribuent à la création et à la transformation de l'ordre social. ${ }^{16} \mathrm{~L}$ 'étude des pratiques administratives néglige souvent la participation des objets matériels ou, plus généralement encore, la matérialité des pratiques. Elle se concentre souvent sur les « réseaux humains » sans prendre en considération la médiation des objets qui constitue les mises en relation et les met en forme. Il est peu de gestes qui composent la texture de ces « réseaux» qui n'aient pas une quelconque matérialité : participation d'un objet, d'un appareil, d'une machine, rapport à des supports de signification sous une forme graphique quelconque. Par ailleurs, tout acte humain est situé et s'inscrit dans un espace matériel qui contribue à le conformer, de même que les objets techniques installés, dans leur fonctionnement même, contribuent aussi à donner forme et sens aux actions humaines. Nous ne sommes pas seulement ici dans une insistance sur l'usage des objets dans les pratiques sociales mais dans une conception du social auquel les objets participent à leur manière. Ainsi, il importe de s'intéresser à la matérialité des pratiques. Mais ce n'est pas seulement parce qu'un discours comme celui de Stalin attire fortement l'attention sur la composante technique indispensable à l'existence de collectifs hiérarchisés comme l'armée ou l'ensemble parti/classe ouvrière (leur cohérence est fournie par la transmission par le télégraphe réel ou par des truchements métaphoriques - qui acquièrent, comme on le verra, une matérialité dans le réel). Les sciences sociales offrent désormais ce terrain technique pour asseoir et penser la stabilité des formes sociales, après avoir longtemps négligé les techniques dans leur pratique ordinaire. Et par surcroît, l'histoire soviétique offre cette dimension particulière que les discours qui servent de référence aux pratiques insistent fortement sur cet aspect technique, plus que dans le monde administratif occidental.

16. Michel Callon et John Law, «L'irruption des non-humains dans les sciences humaines : quelques leçons tirées de la sociologie des sciences et des techniques », in Bénédicte Reynaud, dir., Les limites de la rationalité, vol. 2: Les figures du collectif, Paris, La Découverte, 1997, p. 101. Voir aussi Bruno Latour, L'espoir de Pandore. Pour une version réaliste de l'activité scientifique, Paris, La Découverte, 2001, 347 p. ; id., « Une sociologie sans objet ? Remarques sur l'interobjectivité », Sociologie du Travail, 36, 4, 1994, p. 587-609; Bernard Conein, Nicolas Dodier et Laurent Thévenot, dirs, «Les objets dans l'action. De la maison au laboratoire », Raisons pratiques, 4, 1993,290 p. 
Que les pratiques administratives soient d'abord un rapport à l'espace, à des objets, à des techniques n'a pas échappé à Max Weber qui écrit dans Économie et société :

Il existe pour l'administration bureaucratique des conditions essentiellement liées à la technique des communications. Sa précision exige le chemin de fer, le télégramme, le téléphone, et elle est liée à ceux-ci de façon croissante. Un ordre socialiste ne pourrait rien changer, la question restant de savoir s'il serait dans le cas de créer, comme l'ordre capitaliste, les conditions d'une administration rationnelle $[\ldots]^{17}$.

Pour penser l'administration contemporaine et ses exigences de précision, Weber renvoie aux chemins de fer, au télégraphe et au téléphone, c'est-à-dire à des techniques de parcours de l'espace soit pour les personnes et les objets, soit pour l'information. Il y a d'emblée une question de rapport aux lieux et aux distances, entre le bureau où s'exerce le pouvoir bureaucratique et les lieux de la maîtrise et du contrôle : un rapport à l'espace qui est plus profondément négligé encore dans les sciences sociales que le rapport à la matérialité et aux techniques. Il y a là en puissance une histoire des formes du pouvoir, de la manière dont il circule, s'installe ou se déplace et de la manière dont ses circulations, ses positions et ses déplacements sont pensés. La sociologie d'aujourd'hui complète la série des outils de la précision administrative par des techniques intellectuelles comme les statistiques et les représentations graphiques et aussi par des techniques sociales comme celles de gestion du personnel, visant les salaires, les carrières et les compétences, toutes lourdement équipées d'instruments de plus en plus scientifiques et de machines de plus en plus automatiques.

La portée considérable de l'inscription spatiale des pratiques administratives est bien montrée dans un article de Martine Mespoulet qui décrit la bataille des statisticiens de Saratov, en 1922, pour conserver des locaux séparés du Comité exécutif régional. Le fait que celui-ci cherche à absorber spatialement le bureau de statistique, et ainsi supprimer la marque de son appartenance à une administration centrale, était pour les statisticiens le signe d'une mise en cause de leur indépendance professionnelle. Apparemment, la structure reste inchangée : une «double subordination » à la Direction centrale des statistiques pour appliquer ses directives et au Comité exécutif local dont le bureau est un département. Le conflit montre, en pratique, que c'est l'espace, le cadre immédiat du travail, qui forme la structure. En l'occurrence, le bureau local, non défendu par sa hiérarchie statistique, perd la bataille ${ }^{18}$. Tout l'ensemble des proximités hiérarchiques et de travail en est modifié et le sens des pratiques transformé. Les organisateurs d'entreprise connaissent bien

17. Max Weber, Économie et société, Paris, Pocket, 1995, vol. 1, p. 299.

18. Martine Mespoulet, «Une lutte pour l'autonomie professionnelle : être statisticien dans une région au début des années 1920 », in Jean-Paul Depretto, dir., Pouvoirs et société en Union Soviétique, Paris, Éditions de l'Atelier, 2002, p. 65-88 (ce texte est l'un des très rares à prendre en considération la matérialité du travail administratif). 
les effets de ce phénomène, au moins dès le début du siècle. Les ingénieurs qui s'intéressent au management scientifique veillent soigneusement à régler les voisinages des bureaux d'études avec les ateliers, en compétition avec le voisinage du marché. Ou bien ils s'emploient à régler l'interaction spatiale entre ces mêmes bureaux d'études et les bureaux des méthodes chargés de l'organisation du travail et de la production et donc de la mise en fabrication des pièces conçues dans les bureaux d'études. Les jeux des proximités et des distances ont un effet direct et durable sur l'activité et sur son sens.

Parmi l'immense variété des pratiques administratives (du commandement ou du contrôle à la comptabilité ou à la statistique), j'ai choisi d'illustrer mon propos sur l'importance politique de leur matérialité par trois exemples : celui du dispatching dans l'administration industrielle, celui du téléphone et celui des formalités matérielles du secret. Mais auparavant, j'aborde l'histoire soviétique des systèmes de fiches qui sont une des traduction matérielles de l'idéal télégraphique, mais qui ressortissent aussi à un autre idéal, celui de l'automaticité. Politique de la matérialité, matérialité de la politique.

\section{Une revue de « Technique administrative »}

Au commissariat du peuple à l'Inspection ouvrière et paysanne (RabočeKrest' janskaja Inspekcija - RKI, institution gouvernementale qui forme, à partir de 1923, une seule et même institution avec la Commission centrale de contrôle du parti communiste), une revue se spécialise à partir de 1925 dans l'étude des techniques administratives. Elle a pour titre en russe Tehnika upravlenija, ce que nous traduirions aujourd'hui par La technique de gestion ou bien par La technique de management, et que la revue traduit elle-même par La technique administrative. Elle est publiée par le département nommé Département de technique administrative (pour le coup Otdel administrativnoj tehniki).

La revue se fait la propagatrice de tous les nouveaux systèmes d'organisation et de travail pour le bureau. Elle préconise la rationalisation administrative à la fois dans les entreprises de production et dans l'appareil d'État. Elle vante tout autant l'approche structurelle assistée d'outils de gestion comme l'organigramme que la mécanisation (machines à écrire, à compter, à dicter... et jusqu'aux machines mécanographiques Powers et Hollerith et aux pointeuses) et propage le mobilier de bureau et les systèmes de classement. Plusieurs articles se rapportent à la rédaction des ordres et autres instructions. Tehnika upravlenija incarne ce fait que l'URSS a été un champ bien plus considérable pour l'expérimentation du scientific management industriel et administratif que n'importe quel autre pays au monde. En effet, la rationalisation a été traitée d'emblée comme une affaire d'État tandis que ce même État se donnait à luimême une compétence d'intervention organisatrice jusque dans l'économie, bien au-delà de ses organes administratifs traditionnels. Les "Instituts du travail » prolifèrent sur le territoire soviétique dès les premières années 1920. 
Un Institut national de technique administrative est créé début 1926 sous l'égide de la RKI ${ }^{19}$.

Toutefois, pas plus qu'un discours de Stalin, une telle revue ne dit ce qu'il en est sur place. En général, une revue de ce type, comme n'importe quelle autre en Occident, porte les préoccupations de spécialistes dont le rapport au terrain est à questionner. Leur pratique s'inscrit, ainsi que leur carrière, dans des cadres qui ont leurs propres exigences d'efficacité (ici, cette inspection centrale), exigences différentes des organismes dont ils s'occupent : organes administratifs, entreprises, bureaux... Nous sommes dans une pratique discursive particulière qu'il conviendrait de traiter comme telle et dont il conviendrait d'examiner au cas par cas comment elle s'inscrit dans les activités des praticiens : par quels canaux elle circule, quel type de contrainte elle représente pour les pratiques et sur quels relais, institutionnels ou non, elle s'appuie, quelle assistance elle offre à la verbalisation et à la formalisation des pratiques et en quoi celles-ci de leur côté recourent à cette production discursive et l'insèrent dans leur activité locale.

Un article se distingue pourtant dans le premier numéro de janvier 1925. Il a précisément pour titre «La technique administrative » et est signé d'Elena Rozmirovič, qui est le chef du département de même nom. Elena Rozmirovič (18861953), «vieille bolchevique», personnellement connue de Lenin, membre du Présidium de la RKI, est une des très rares femmes qui émerge entre les deux guerres, avec un peu plus tard la Britannique Mary Parker Follett, dans le milieu exclusivement masculin des penseurs de l'organisation et de l'administration (auxquelles on pourrait ajouter toutefois les très tayloriennes Christine Frederick, américaine, et Paulette Bernège, française, qui se spécialisent dans le travail domestique). On trouve dans cet article une autre formulation de l'idéal télégraphique de gouvernement que celle de Stalin ${ }^{20}$. L'intérêt de ce texte est qu'il propose une consistance technique à ce qui n'est que réseaux d'appareils, antennes et courroies de transmission métaphoriques dans le discours de Stalin, s'agissant du lien de commandement entre le parti et la «classe ouvrière », son «armée ». Rozmirovič, son département et la revue Tehnika upravlenija connectent l'idéal technoadministratif de la politique porté par Lenin et Stalin - nous ne sommes pas très éloignés de l'idéal industriel de l'État porté par les Saint-Simoniens - , d'une part,

19. Sans citer d'autres textes d'époque et parmi une littérature proliférante, voir Kendall E. Bailes, « Aleksei Gastev and the Soviet controversy over Taylorism, 1918-1924 », Soviet Studies, 29, 1977, p. 373-394 ; Eduard B. Koritskij, Iurii A. Lavrikov, Problemy razvitija teorii upravlenija socialističeskim proizvodstvom (Problèmes de développement de la théorie de gestion de la production socialiste), Leningrad, Izd-vo LGU, 1982, 207 p. (rééd. 1989, 271 p.) Mark R. Beissinger, Scientific management, socialist discipline, and Soviet power, Cambridge (Mass.), Harvard University Press, 1988, 376 p. Robert Linhart, Lénine, les paysans, Taylor (Paris, Seuil, 1976, 172 p.) est surtout une lecture des textes de Lenin.

20. Sur Elena Rozmirovič, voir aussi Eduard B. Korickij, Jurij A. Lavrikov, Alim M. Omarov, Sovetskaja upravlenčeskaja mysl' 20-h godov. Kratkij imennoj spravočnik (La pensée managériale soviétique dans les années 1920. Petit annuaire), Moscou, Ekonomika, 1990, 231 p. et Eduard Korickij, $U$ istokov NOT. Zabytye diskussii i nerealizovannye idei (Aux sources de I'OST. Discussions oubliées et idées non réalisées), Leningrad, Izd-vo Leningradskogo Universiteta, 1990, $334 \mathrm{p}$ 
avec les recherches internationales en matière de théories et de machines administratives et, d'autre part, avec les efforts pour réformer l'administration du parti, du gouvernement et des entreprises. Nous ne sommes plus dans le simple télégraphe qui transmet les ordres au sein de l'armée, mais dans un complexe matériel et humain de plus en plus intense, spécialisé et équipé de techniques qui ne manquent pourtant pas de se penser dans l'ordre de la transmission.

\section{Automatiser par les normes de travail et par les fiches}

La tâche majeure consiste en «l'amélioration de la technique de l'appareil d'État » (expression que l'on trouve aussi sous la plume de Kujbyšev, alors commissaire à la $\mathrm{RKI}^{21}$ ).

D'une part, l'analogie est tissée de façon répétitive entre processus de production d'objets matériels et processus de traitement administratif. Dans la production, on sait comment étudier le travail. Le taylorisme y donne la norme de l'établissement des normes. Elle peut servir de modèle. Dans le travail administratif, il faut également tout saisir par la norme. Lenin met son poids à plusieurs reprises pour aller dans ce sens et jusque parmi les dernières de ses lettres. Il écrit ainsi à l'un des chefs adjoints de la RKI :

J'estime que nous devons élaborer une normalisation du travail de bureau et l'appliquer ensuite partout. C'est la chose la plus importante. [...] Le principal, ce sont les normes (c'est-à-dire combien de personnes pour telle somme de travail). Après quoi nous obligerons notre Office central de statistique à travailler également ${ }^{22}$.

D'autre part, la fin proposée consiste à réduire la pratique administrative à de simples «mécanismes ». Les normes de travail sont la première condition de cette mécanisation :

L'ensemble de ces normes de travail particulières, élaborées, automatisées, et leur conditionnement serré dans le temps, dans l'espace et les unes par rapport aux autres créent ensuite un système précis de gestion de tout le processus de travail. Ce système se présente ainsi comme un système d'actions humaines liées entre elles, un mécanisme précis - une machine où chaque petit rouage est à sa place et possède une signification précise ${ }^{23}$.

Nous avons une notion d'administration comme système d'actions étroitement normées. Où est la part de la matérialité ? Dans les fiches.

21. Valerian Kujbyšev, «Zadači CKK i RKI po racionalizacii gosapparata v plane rabot RKI na 1925-26 g. » (Les tâches de la CCC - RKI pour la rationalisation de l'appareil d'État dans le plan de travail de la RKI pour 1925-26), Tehnika upravleniia, 6, 1925, p. 3-14.

22. V. I. Lénine, Euvres, t. 36, 1959, p. 595 (Lettre à V. A. Avanesov, le 1er septembre 1922).

23. Elena Rozmirovič, «Tehnika upravlenija » (La technique administrative), Tehnika upravlenija, 1, janvier 1925, p. 7. 
Pour instaurer ce mécanisme et même cet automatisme des processus administratifs, Elena Rozmirovič ne propose en effet pas seulement les normes régulées mais « les systèmes de fiches » « à l'européenne ».

\section{L'agency des « systèmes de fiches »}

Ici, il est important de le marquer, nous n'avons plus affaire à un taylorisme de base mais aux méthodes élaborées dans le sillage du scientific management et qui provoquent une « révolution invisible » dans les bureaux, ainsi que la nomme Delphine Gardey. Des revues américaines comme System propagent une nouvelle organisation du travail dans le bureau. Le centre de l'activité du bureau est la mise des écritures en fiches, outillée par des machines à reproduire, à écrire ou à compter. Étrangement pour nos esprits formés à la fin du XX' siècle, l'usage de fiches et de feuillets mobiles est alors une nouveauté dans les bureaux. Ils remplacent progressivement les gros registres peu maniables et les recueils de correspondance difficilement indexés. Les «systèmes » sont les dispositifs très matériels de classement de ces fiches: meubles, kyrielle de petites dispositions de couleurs, de marques, d'onglets, de relevé des déplacements ${ }^{24}$. «La mécanisation à l'aide d'un système de fiches » est selon Rozmirovič « la forme de rationalisation la plus populaire et la plus appliquée actuellement » pour « la gestion et la technique générale de l'appareil d'État». Ces systèmes sont d'abord développés aux États-Unis avant d'être publiés en Europe. C'est probablement d'Europe que vient l'information des administrateurs de la RKI qui ne citent cependant pas leurs sources précises.

À plus long terme encore, cette «mécanisation » conduit, selon Rozmirovič, à une véritable « automatisation » de la gestion reposant sur la fiche. Nous sommes là au plus haut de la démonstration et il ne faut rien moins qu'une phrase immense de 16 lignes pour en exposer la thèse. Je la découperai ici pour la rendre intelligible, en proposant une traduction proche de la paraphrase. La fiche se manifeste grâce à une multitude de signes prévus par le « système », couleurs, formes, onglets, arrangement physique et enregistrement de ses mouvements. Le contenu de la fiche est aussi réparti dans une série réglée d'autres fiches. Cet ensemble réglé de signes matériels et d'objets prédétermine la volonté et l'action de l'administrateur tout autant que des employés dans l'administration concernée. Il les relie en un seul «système». En même temps, une fois que l'action de l'administrateur s'est produite ou qu'il a délivré son instruction, cet ensemble crée un mouvement qui reçoit un cours automatique indépendant de sa volonté. Ce mouvement, ayant trouvé son expression matérielle précise dans la fiche, engage une chaîne coordonnée et indissoluble d'actions de la part des autres personnes. Cette chaîne court

24. Delphine Gardey, La dactylographe et l'expéditionnaire. Histoire des employés de bureau, 1890-1930, Paris, Belin, 2001, p. 127-138. Pour les États-Unis, cette « révolution administrative » avait déjà été présentée par JoAnne Yates, Control through communication. The rise of system in American management, Baltimore, Johns Hopkins University Press, $1989,368 \mathrm{p}$. 
d'une partie à l'autre de l'administration jusqu'à l'obtention de l'effet recherché. Celui-ci est d'ailleurs contrôlable par d'autres personnes encore, liées entre elles par ces mêmes signes matériels comme les maillons d'une même chaîne (les italiques sont dans le texte). Dans cette exaltation de l'agency du système de fiches (qui « détermine » l'action), on n'est pas loin de la description d'un réseau socio-technique où le sens se transforme d'une traduction à l'autre au gré des passages par les nouds humains et non humains ${ }^{25}$. Le vocabulaire dominant ici est toutefois celui de la chaîne et c'est bien une référence à la chaîne de Ford qui clôt le texte :

La logique de fer de ce convoyeur crée une coordination objectivement inévitable des personnes et des choses dans le processus de production. Créer une même coordination objective entre les gens et les choses, c'est ce que nous voulons faire dans le processus de gestion [...]. Alors, chaque ouvrier pourrait effectivement gérer, presser le bouton approprié de cet appareil automatique en provoquant ainsi le mouvement automatique de tout le convoyeur (lenta) de gestion.

La chaîne des fiches permet à la cuisinière de gérer l'État. Même si cette «mécanisation de la gestion » est présentée comme un « idéal », on voit que, pour Elena Rozmirovič, elle seule permettrait la participation active des travailleurs au travail administratif et au contrôle de l'État, rappel d'un des objectifs majeurs des bolcheviks. Le « système de fiches » accomplit la révolution tout court, la révolution politique.

Par ailleurs, cette pensée étonnante pour nous d'un automatisme qui repose sur les seules fiches ne devrait pas provoquer le sourire. Chez Ford aussi, la fiche « automatise » la gestion. À Detroit, explique une revue française, « la progression de chaque ouvrier est attentivement suivie par la Direction elle-même qui tient à jour des fiches individuelles ou livrets où figurent tous les renseignements voulus ». Si la direction n'est pas informée en temps voulu de changements qui devraient normalement intervenir dans la progression du salaire ou de la qualification, grâce aux fiches, elle «s'en aperçoit automatiquement et fait enquête ${ }^{26}$. Les fiches, par leur seule existence, déclenchent une réaction (humaine) automatique. Nous sommes à une époque datée de l'histoire de l'automatisme.

\section{Automates humains}

Le rôle de l'humain n'est plus que celui de régulateur automate. Nous participons, en URSS et aux États-Unis, de la même pensée de l'automatisme humain, probablement liée elle-même à un vocabulaire psychologique partagé à travers les frontières.

\section{Voir M. Callon, op. cit.}

26. Art. anonyme, «Collaboration, cohésion », Bulletin officiel de la Chambre syndicale des constructeurs d'automobiles, 60, août 1917, p. 2. 
Le travail des agents humains est en effet conçu, lui aussi, comme un automatisme. Ainsi, Rozmirovič écrit-elle un peu plus tard :

La mécanisation du processus de production dans le cadre de l'entreprise prise comme un tout reconfigure fondamentalement la gestion en la transformant d'un commandement (rasporjaditel'stvo) des hommes en une régulation (regulirovanie) des processus objectifs et matériels où l'ouvrier ne figure précisément que comme un mécanisme de contrôle précis.

En d'autres termes, la production est de plus en plus prise dans une matérialité technique. Ses problèmes se résument de plus en plus à de la régulation à laquelle les ouvriers participent comme instruments de contrôle qui réagissent aux variations des machines. Les principes s'en étendent à l'administration. Dès lors, dit en effet Rozmirovič, « la régulation du processus objectif et matériel, à la différence du commandement des hommes, est assurée par l'intermédiaire d'instruments de régulation et revêt une forme objectale et matérielle » et « la construction d'un appareil de gestion $[\ldots]$ se transforme en un simple problème technique. $»^{27}$

Considérer l'humain comme un automate (en référence directe aux machines automatiques comme les automates de Vaucanson) est une tradition dans la psychologie contemporaine. Ainsi l'œuvre qui rend Pierre Janet célèbre traite de l'automatisme considéré comme «activité humaine dans ses formes les plus simples, les plus rudimentaires » opposée aux «formes les plus élevées », « la volonté, la résolution, le libre arbitre ${ }^{28}$. La première étude de psychologie du leadership aux États-Unis, œuvre d'un célèbre psychologue de l'enfance adaptateur $\mathrm{du}$ test de Binet-Simon, Lewis Terman, investit la distinction de ces «formes » psychologiques dans différents types humains et oppose le leader à l'automaton ${ }^{29}$. Nous sommes bien ici dans un mode de pensée qui circule et traverse les frontières. Les penseurs soviétiques de l'administration ne sont pas coupés des milieux européens dans les années 1920 et les penseurs de l'administration en général n'ignorent pas les travaux des psychologues ${ }^{30}$.

Les notations présentes ne sont assurément que des pistes de travail : il conviendrait de détecter les connexions précises, de localiser les occurrences dans un corpus étendu en durée et en espace, de relever la circulation des œuvres et les lectures, de

27. Elena Rozmirovič, «Metodiki i metodologija » (Méthodes et méthodologie), Tehnika upravlenija, 7, juillet 1926, p. 16-17.

28. Pierre Janet, L'automatisme psychologique. Essai de psychologie expérimentale sur les formes inférieures de l'activité mentale, Paris, Odile Jacob, 1998, p. 33 (1re éd. Paris, Alcan, 1889). Janet est une source d'inspiration majeure pour le fondateur de l'école américaine des « relations humaines » dans l'industrie : voir Elton Mayo, The human problems of an industrial civilization, New York, Macmillan, 1933, 194 p. et id., Some notes on the psychology of Pierre Janet, Cambridge (Mass.), Harvard University Press, 1948, 132 p.

29. Lewis M. Terman, « A preliminary study in the psychology and pedagogy of leadership », Pedagogical Seminar, 11, 1904, p. 413-451. Voir encore H. Stafford Hatfield, Automaton or the future of the mechanical man, Londres, Kegan Paul, Trench, Trubner \& Co, 1928, 100 p.

30. La RKI traduit ainsi des textes du 2 e Congrès international de sciences administratives tenu en 1923 à Bruxelles, comme Pol Otle [Paul Otlet], Rukovodstvo i administrirovanie (Commandement et administration bureaucratique), Moscou, NKRKI (Serija 'Rukovodstva'), 1924, 85 p. 
suivre aussi les personnes à travers les frontières, sans exclure la possibilité d'inventions locales simultanées issues de situations homologues, comme en matière purement technique, et surtout de scruter les pratiques sur les lieux de travail.

En tout cas, cette conception technique, propagée par Rozmirovič, d'une administration fondée sur un mécanisme matériel et humain automatique nous emporte loin de la politique, au sens de la manifestation ménagée de la mésentente des parties dans le jeu social. La cuisinière qui gère l'État n'a qu'à appuyer sur un bouton pour entraîner la chaîne administrative. Aux temps à venir de l'idéal passé dans le réel, sa poussée sur le bouton sera, par un fait d'ontologie, forcément la bonne décision. Ainsi dans la pensée administrative soviétique, nous sommes endeçà d'une théorie de la décision dans une organisation non démocratique comme il commence à s'en réfléchir pour l'entreprise et l'armée dans les pays capitalistes. Bien sûr, l'Union soviétique ne saurait être considérée par ses chefs comme non démocratique. La décision n'est jamais en question. En effet, ce qui est ici pensé, c'est ce qui doit advenir aux lendemains chantants tandis que le présent est la délivrance de la ligne forcément juste par les organes supérieurs du parti. Au XII congrès d'avril 1923, Stalin introduit son propos sur les cadres obéissants par: «Quand la ligne politique juste est donnée, il faut choisir les cadres... $»^{31}$.

Nous sommes entièrement dans des pratiques administratives pensées comme il se doit comme exécution, dans le cadre d'une politique qui consiste activement à chasser toute politique.

\section{Où sont les fichiers ?}

On peut se poser la question de savoir où, en dehors de ces formulations idéalisées, ces systèmes de fiches sont mis en place effectivement. Elena Rozmirovič évoque nommément, dans son article de janvier 1925, seulement trois administrations dans lesquelles l'application de ces méthodes a permis de diminuer de façon radicale le temps et la peine des employés et du public et les dépenses de l'État: la Cour suprême, les chemins de fer et les caisses d'assurance, mais elle ne donne pas de renseignements plus précis.

Le « système de fiches » semble encore atteindre, dès 1923, l'organe qui est déjà le centre du pouvoir, le Secrétariat général du parti. Molotov recommande à Stalin un jeune secrétaire de l'organisme qu'il préside, l'Orgbjuro, le bureau d'organisation du Comité central, Boris Bažanov, pour ses talents d'organisateur du travail de bureau. À en croire le témoignage de Bažanov lui-même, il introduit en 1923 le système de fiches à l' Orgbjuro puis au Secrétariat général où il devient secrétaire du Bureau politique (Politbjuro) en août 1923. Voici son récit :

La mer de papier dans laquelle est noyée l'Orgbjuro est un tel fouillis qu'on ne peut rien trouver ; les documents sont enregistrés d'après des méthodes antédilu- 
viennes d'entrées et de sorties. Lorsque le secrétaire du Comité central a besoin d'un renseignement ou d'un document des archives, on se met à chercher pendant des heures dans l'océan d'archives. Je vois que cette organisation ne vaut rien ; je la balaie et établis plusieurs cartothèques [sic. La seule traduction correcte de kartoteka est fichier] où chaque document est inscrit sur trois index alphabétiques différents. Petit à petit, chaque chose prend sa place. Au bout de deux ou trois mois, le papier ou le renseignement réclamé par le secrétaire du Comité central lui est remis une minute après. Les sections du Comité central qui considéraient auparavant que cela ne servait à rien de s'adresser au secrétariat de l'Orgbjuro sont émerveillées par la rapidité avec laquelle tout se fait sur-le-champ. Molotov est extrêmement satisfait et ne cesse de chanter mes louanges.

Bažanov arrive donc en août chez Stalin, au Politbjuro : « La pagaille et le chaos sont encore plus grands ici qu'à l'Orgbjuro: les montagnes de paperasses sont beaucoup plus grandes et entassées dans un ordre incompréhensible. On réussit rarement à dénicher un papier ou un renseignement ». Comme c'est habituel dans ce cas, tout repose sur la mémoire exceptionnelle d'un collaborateur, en l'occurrence d'une collaboratrice. «Je m'applique à tout réorganiser comme à l'Orgbjuro, à l'aide de fichiers et d'index » et les résultats de la réforme sont «miraculeux » : «Deux ou trois mois plus tard, tout est rentré dans l'ordre et on trouve chaque document instantanément.» ${ }^{32}$ Bažanov, ancien élève de l'École technique supérieure de Moscou, ne dit pas d'où il tient sa maîtrise du fichier ni à quoi ou à qui il doit les connaissances grâce auxquelles il opère la réforme qu'il s'attribue sans que nous puissions vérifier la véracité de cette attribution. Est-ce à elle que Stalin est redevable du sobriquet de «camarade Kartotekov » (le camarade aux fichiers) dont, paraît-il, il était affublé alors, ou bien à ces autres fichiers encore plus secrets dans lesquels il accumule les notations sur les personnes auxquelles il a affaire, et dont Bažanov ne dit rien ${ }^{33}$ ?

L'histoire de cette réforme du travail administratif reste à faire, ainsi que celle de son rapport à la prolifération bureaucratique dont l'Union soviétique est le siège. Un des débats les plus intéressants du colloque sur le NKVD, organisé en l'an 2000 par Andrea Graziosi, Terry Martin et Jutta Sherrer, a porté sur les différents fichiers utilisés au cours des répressions successives, leur caractère plus ou moins systématique ou rudimentaire, y compris sur le plan de leur matérialité. Il en ressortait que, même dans la police politique où la récapitulation des personnes est une opération cardinale, le fichier est loin d'être généralisé à la haute époque des purges des années 1937 et 1938 qui sont largement dépendantes de listes constituées, sans aucune méthode, sur plusieurs années ${ }^{34}$. La modernité des fichiers n'était pas une nécessité absolue pour cette vague moderne de répression de masse. Par ailleurs, la

32. Boris Bajanov, Bajanov révèle Staline. Souvenirs d'un ancien secrétaire de Staline, Paris, Gallimard, 1977, p. 36 et 48-49. Ce livre reste un des témoignages les plus précis de l'activité de Stalin au début des années 1920.

33. Michael Voslensky, La Nomenklatura. Les privilégiés en URSS, Paris, Belfond, 1980, p. 77.

34. Andrea Graziosi, Terry Martin et Jutta Scherrer, dirs, « La police politique en Union soviétique, 1918-1953 », Cahiers du Monde russe, 42, 2-4, avril-décembre 2001. 
pénurie de moyens d'un État somme toute pauvre, qui se manifeste entre autres par le manque chronique de papier, n'est certainement pas à négliger ${ }^{35}$.

Quoi qu'il en soit de la mise en place de systèmes de fiches dans l'administration et de son effet sur la pratique administrative dans ses opérations les plus essentielles comme la catégorisation des cibles de l'administration, l'idéal automatique est en échec. En 1930, Elena Rozmirovič, proche de Buharin qui est lui-même de plus en plus écarté de positions actives, est l'objet d'attaques. Elle a participé à la conception fonctionnelle de l'organisation de l'économie qui s'avère excessivement compliquée à mettre en place et aggrave le chaos dans lequel l'industrie soviétique est plongée ${ }^{36}$. Les critiques visant Rozmirovič mettent en cause son idéal mécanique, « techniciste étroit », du social. Elle est d'ailleurs proche, en idées comme par les alliances internes aux milieux de la rationalisation, d'Aleksej Gastev (18821941), le « barde prolétarien de l'ère mécanique » qui conçoit les ouvriers comme devant vivre et travailler au rythme des machines : une vision commune poussée chez eux beaucoup plus loin qu'elle ne l'est chez les ingénieurs occidentaux, même les tayloriens farouches ${ }^{37}$. Les dirigeants staliniens, comme Ordžonikidze, qui passe de la tête de la RKI à la direction de l'économie à la fin de 1930, et surtout Andreev qui le remplace à la RKI, prennent leurs distances par rapport au mouvement de rationalisation. Elena Rozmirovič ne dispose pas des bons alliés au bon moment. Elle est écartée de ses positions dirigeantes dans la RKI en 1931. Son Institut de technique administrative est fermé en $1932^{38}$.

\section{L'américanisme et l'idéal automatique à l'épreuve}

L'idéal automatique du système de fiches conférait de la matérialité à l'idéal télégraphique de gouvernement énoncé par Stalin en 1923. Nous en sommes surtout restés, dans leur considération, aux aléas des pratiques discursives. De plus, n'ont été évoquées là que quelques bribes de ce qui compose une culture beaucoup plus large de confiance dans la capacité des sciences pratiques à résoudre les problèmes posés par la société. Si l'on se tourne vers des témoignages plus proches de la pratique (qui ne seront pas encore des témoignages directs), on peut tenter de saisir comment cette culture informe les manières de faire.

En 1926, une délégation de dirigeants et d'ouvriers de Ford visite l'Union soviétique pour y examiner et expertiser la production et l'usage des tracteurs Fordson.

35. Sur la pénurie de papier et ses effets, voir Nathalie Moine, «Le système des passeports à l'époque stalinienne. De la purge des grandes villes au morcellement du territoire, 1932-1953 », Revue d'Histoire moderne et contemporaine, 50, 1, janv.-mars 2003, p. 145-169, ici p. 162.

36. David R. Shearer, Industry, state, and society in Stalin's Russia, 1926-1934, IthacaLondres, Cornell University Press, 1996, p. 159 en particulier.

37. Kurt Johansson, Aleksej Gastev. Proletarian bard of the machine age, Stockholm, Almqvist et Wiksell International, 1983, 169 p. (le roman de Zamjatin, Nous autres, est la description effrayée de l'humanité future engendrée par un tel système).

38. M. Beissinger, op. cit., p. 117-119. 
Ces tracteurs y sont importés depuis 1924. Ils sont en 1926 au nombre de 20000. Des écoles de conduite et de réparation ainsi que des ateliers spécialisés sont organisées dans les campagnes. L'usine Putilov Rouge de Leningrad commence à en produire au rythme faramineux de 2 par mois. Avant d'entreprendre sa tournée, la délégation est reçue à Moscou où on lui montre avec un enthousiasme débordant les résultats obtenus qui laissent présager un succès massif. Dans leur rapport (de 266 pages), les délégués de Ford indiquent que les Soviétiques « sont devenus dingues des graphiques, diagrammes et autres tableaux mathématiques ». Les courbes de réparation, d'activité des écoles et d'inspection dans les régions affichent des performances extraordinaires. Sur place, les hommes de Ford cherchent cependant en vain les écoles et les ateliers qui correspondent à ce qu'indiquent les graphiques. En Ukraine, où les tracteurs sont le plus utilisés, on peine à dénicher, écrivent-ils, « un seul atelier de réparation de Fordson digne de ce nom ». Un seul, à Rostov, trouve grâce à leurs yeux. Tout ceci n'affectait en rien la réalité triomphale des graphiques exposés à Moscou ${ }^{39}$.

Un autre exemple, celui de l'irruption du dispatching dans la gestion de l'industrie à la fin des années 1930, montre plus fortement en acte l'idéal automatique ou, autrement dit, comment l'idéal, convoqué comme une référence directrice de leur action par les administrateurs praticiens, opère effectivement dans le réel. Nous n'aurons ainsi pas seulement des acteurs matériels, humains, spatiaux, mais aussi des acteurs idéaux que seule toutefois leur attestation par des inscriptions au sein des pratiques, ici des pratiques administratives, permet de saisir comme tels. Ni la proposition de Stalin, même appuyée en 1923 sur sa position déjà extrêmement puissante de secrétaire général, ni celle de Rozmirovič, appuyée sur les relais des entreprises gouvernementales de rationalisation, qui se révèlent fragiles à l'usage, n'auraient pu constituer à elles seules les idéaux télégraphique et automatique en acteurs dans le réel. L'idéal comme acteur de la pratique ne saurait être saisi que dans ses actualisations. L'idéal - ou l'utopie - comme objet de pratique discursive ne dit rien de sa présence dans l'action.

\section{Le dispatching}

Le dispatching et le dispatcher sont des figures plus familières dans le paysage russe que dans le paysage français. Le dispatching désigne en Russie tout ce qui est répartition du travail dans les entreprises ou distribution des opérations dans les systèmes d'urgence. En France, c'est seulement une métaphore de la répartition ou une étroite figure professionnelle de la distribution de l'électricité. En toute généralité, il s'agit d'une technique de régulation des opérations ou encore de répartition et de coordination des tâches. Il est progressivement introduit dans le système industriel soviétique au cours des années 1930. Or le dispatching est une variation sur le système de fiches,

39. Mira Wilkins, Frank Ernest Hill, American business abroad. Ford on six continents, Detroit, Wayne State University Press, 1964, p. 213-214. 
un peu plus compliquée puisqu'aux fiches sont adjoints des systèmes de communication des ordres qui peuvent être simplement le téléphone mais aussi des systèmes lumineux gérés par des commutateurs. L'inclusion de ces questions de direction industrielle dans le présent article suppose d'accepter un langage un peu spécialisé par rapport au vocabulaire habituel de la pratique administrative.

Ce procédé est d'abord expérimenté aux États-Unis à la fin du XIXe siècle dans les chemins de fer, puis dans la production et la distribution de l'électricité. La méthode est transférée à l'industrie dans le sillage de l'investissement des opérations industrielles par l'esprit du management scientifique taylorien. Les bolcheviks ne pouvaient pas manquer de lui être sensibles. Les manuels d'organisation américains le décrivent en effet comme ce qui ordonne la mise en œuvre du plan. Cette technique peut être plus ou moins formalisée. Elle recourt parfois à une instrumentation mathématique assez poussée. Il s'agit en effet d'analyser les opérations et de les répartir en termes de durée et de charge pour les hommes et pour les installations entre les différents secteurs et les différents équipements techniques.

Sur le plan matériel, le système se présente, on l'a vu, comme un ensemble de fiches très élaboré et articulé. Nous retrouvons là la même matérialité révolutionnaire des pratiques administratives que celle dont Rozmirovič parlait. Dans le bureau du dispatcher, ces fiches sont disposées sur des tableaux muraux. Ces tableaux font ainsi apparaitre les modalités de l'engagement des installations, des équipes et des machines. Sur place, dans les ateliers, auprès des machines, des tableaux correspondants reproduisent la version locale de ce qu' affichent ceux du poste central de dispatching. La coordination entre les tableaux est assurée par des dispositifs d'information et de communication qui permettent le pilotage des installations autant que possible en temps réel (soit par le déplacement d'agents spécialisés, soit au moyen du téléphone ou de dispositifs spéciaux de signalisation électrique).

Une partie des opérations que réalise le dispatcher peut se faire dans sa cabine. D'autres opérations supposent au contraire le déplacement sur place pour se rendre compte en personne des problèmes de coordination et pour les interpréter de façon pertinente. En d'autres termes, il s'agit d'un système d'action et de contrôle à distance qui ne saurait se passer entièrement d'information en présence.

Le mouvement pour introduire le dispatching dans l'industrie soviétique est engagé en 1932 et 1933. Il correspond à une période particulière des modes managériales en Union soviétique. À ce moment, «l'organisation fonctionnelle» est soumise à la critique alors qu'elle avait constitué la panacée dans la seconde moitié des années 1920. L'organisation fonctionnelle est une autre importation des ÉtatsUnis. Pour résumer, elle consiste à introduire des structures parallèles à la ligne hiérarchique verticale dominante et consacrées à des thèmes particuliers avec fonction de conseil ou de service plutôt que de commandement : finance, comptabilité, rationalisation, recherche, approvisionnement... ${ }^{40}$ Par exemple, un département de

40. L'étude classique est celle d'Alfred D. Chandler, Jr, La main visible des managers. Une analyse historique, Paris, Economica, 1988, 635 p. (éd. originale: The visible hand. The managerial revolution in American business, Cambridge (Mass.), Belknap Press of Harvard University Press, 1977). 
rationalisation peut exister au niveau central d'une entreprise et avoir des relations directes avec tous les bureaux de rationalisation organisés à chacun des autres niveaux hiérarchiques, ceux du département, de l'atelier, etc. Cette organisation est développée dans toute l'économie soviétique dans le courant des années 1920. Elle est remise en cause au début des années 1930 : pour poursuivre l'exemple, le département de rationalisation est supprimé au centre des entreprises et les différents bureaux ne peuvent plus entrer en relation directe les uns avec les autres. L'introduction du dispatching est une parmi toutes les mesures qui manifestent un resserrement sur la hiérarchie verticale.

La région de Leningrad étant considérée comme le «maillon principal de l'industrie lourde », elle est instituée comme le «laboratoire » de l'Union soviétique pour l'introduction du dispatching ${ }^{41}$. En 1934, 30 entreprises sont concernées par cette opération, la plupart fabriquant du matériel électrique, les autres étant des usines métallurgiques et mécaniques.

Le dispatching fait l'objet d'une généralisation dans l'appareil de gestion industrielle à la fin de 1937, c'est-à-dire au pic de la terreur répressive qui s'abat sur le pays de 1937 à $1938^{42}$. Il est mis en place dans les directions centrales du commissariat du peuple à l'Industrie lourde (Narodnyj Komissariat Tjaželoj Promyšlennosti NKTP) et doit réorganiser les usines. Il est considéré comme la solution miracle pour le règlement des problèmes de l'administration industrielle. Le NKTP est alors le principal ministère industriel. Toutes ses directions centrales, chacune étant chargée d'une branche différente (charbon, électricité, production métallurgique, or, etc.), sont réorganisées de la même façon. Il leur est ajouté deux départements, un département technique et un département de commandement de la production (proizvodstvenno-rasporjaditel'nyj otdel). Ce dernier département est peuplé de dispatchers qui sont érigés en «figures centrales » de cette réforme ${ }^{43}$. Ces spécialistes sont de jeunes ingénieurs nouvellement promus à la faveur des grandes purges qui continuent d'éclaircir férocement les rangs des directions (un rapport qui traite du dispatching, adressé mi-1938 à Kaganovič, le commissaire du peuple, par le chef du groupe central de contrôle et d'inspection du NKTP, note par exemple que dans une des directions opèrent encore 4 saboteurs parmi les directeurs et 15 parmi les ingénieurs en chef et que, dans les usines, le remplacement des saboteurs progresse extraordinairement lentement). La généralisation du dispatching est donc liée à la grande répression et au renouvellement stalinien des cadres.

Les dispatchers sont envoyés dans les usines de leur direction pour se familiariser avec elles et y organiser le dispatching. Leur appartenance à l'appareil admi-

41. G. V. Cihockij, « Dispečerizacija v tjaželoj promyšlennosti » (Le dispatching dans l'industrie lourde), in N. L. Zajcev, dir., Dispečerizacija v leningradskoj promyšlennosti (Le dispatching dans l'industrie de Leningrad), Leningrad, Izd. informacionno-izdatel'sk. bjuro LOUMP, 1935, p. 54-72.

42. M. Beissinger, op. cit., p. 145.

43. Rossijskij Gosudarstvennyj Arhiv Ekonomiki (Archives d'État de l'économie), 7297/28/ 40a/8, rapport à L. Kaganovič, commissaire du peuple à l'Industrie lourde, de Nikolaev, chef du groupe central de contrôle et d'inspection du NKTP, s.d. (après mai 1938). 
nistratif central est censée rapprocher les directions ministérielles des entreprises et renforcer la «direction opérationnelle » aux deux niveaux du ministère et des usines. La technicité du dispatching et sa matérialité faite de fiches et de systèmes de communication sont supposées régler de nombreux problèmes apparus au cours des premiers plans quinquennaux. Le principal est l'extrême difficulté de coordonner la production au sein des entreprises en raison de l'irrégularité chronique de l'approvisionnement. L'ouvrage de 1935 sur la mise en place du dispatching dans la région de Leningrad indique, dans les usines où le dispatching fonctionne le mieux, une forte diminution des communications écrites entre ateliers pour régler des affaires d'approvisionnement. Il s'y ajouterait une réduction des prix de revient, une diminution des à-coups, des arrêts de la production et du nombre de produits qui restent non finis dans les aires de stockage. Ce n'est ici pourtant que le témoignage d'une proclamation : l'enquête historique qui étudierait en détail ce qui se produit dans les entreprises n'est pas faite.

On pourrait contester le fait de compter le dispatching parmi les techniques administratives. Il s'agit pourtant de pure administration opérationnelle de la production, sans aucune métaphore. Le dispatching est pensé dans le même univers que celui de l'organisation formelle des activités et recourt à sa manière à des « systèmes de fichiers ». Enfin, il participe pleinement à la continuité de l'espace de prescription que j'évoquais plus haut. Par rapport à une histoire classique de l'administration, en Union soviétique, après la fin de la NEP, l'unité économique de base est prise dans le même tissu administratif que les organismes gouvernementaux et que le parti unique de gouvernement : originalité de cette dictature.

\section{Des intensifications techniques}

L'idée même d'un dispatching et ses techniques sont importées d'Amérique. Pourtant, à étudier non pas comment idée et techniques se sont diffusées mais comment le dispatching a pris forme et a été redéfini en Union soviétique, on fait apparaître des configurations particulières où les sources américaines sont difficiles à reconnaître et à isoler.

Tout d'abord, le dispatching est célébré comme un «système de direction de la production centralisé, complexe et opérationnel ». Les adjectifs sont là pour indiquer que le dispatching intervient comme une solution alternative à l'organisation fonctionnelle. Celle-ci a été condamnée au début des années 1930 et proprement «liquidée » en $1934^{44}$. À partir de ce moment, le dispatching occupe une place centrale dans la définition de l'organisation de la production alors qu'il n'est indiqué, dans la littérature d'organisation américaine, que comme un système d'ordonnancement des activités et de circulation des produits dans le cours de leur

44. David R. Shearer, «Factories within factories. Changes in the structure of work and management in Soviet machine building factories, 1926-1934 », in William G. Rosenberg, Lewis H. Siegelbaum, dirs, Social dimensions of Soviet industrialization, Bloomington, Indiana University Press, 1993, p. 193-222. 
élaboration, essentiel sans doute mais pris dans tout un ensemble d'autres dispositifs ${ }^{45}$. Le dispatcher en chef des usines soviétiques est placé immédiatement sous le directeur technique qui est adjoint au directeur d'usine. Il est en charge de la direction de la production et absorbe les services de planification qui étaient les principaux services fonctionnels. « Avec le dispatching, il n'y a plus de commandement latéral (komandovanie so storony, c'est-à-dire fonctionnel) des ateliers » : la subordination est clairement verticale, les ordres tombent en cascade et la discipline est heureusement renforcée ${ }^{46}$.

En second lieu, ce qui contribue à la singularité soviétique est la forte valorisation du fait que le dispatching est une solution technique, très matérielle, à des problèmes d'organisation et de coordination. Contre la mauvaise solution qu'est l'organisation fonctionnelle fondée sur des formes, la confiance ici est fondée sur la matérialité des systèmes de fiches et - c'est au moins souhaité - sur tout un appareillage de commutateurs et de signaux qui transmettent les ordres selon les lignes définies au centre. On conçoit alors que, dans l'atmosphère répressive de 1937-1938, le dispatching soit un recours contre le « bureaucratisme ». Un des principaux travers de celuici est précisément la direction à distance que le dispatching entend traiter de façon nouvelle, en l'occurrence par une révolution technique ${ }^{47}$. Mais ce qui fait la particularité du dispositif mis en place en 1937 est que le dispatching n'est pas là une nouvelle forme de l'intervention classique du centre dans les usines sur le mode du coup de poing ou du commando. Les dispatchers des directions centrales doivent rester plusieurs mois sur place pour connaître de près les entreprises. La longueur du déplacement est le garant d'une connaissance utile autant à l'entreprise qu'au centre. Elle est un procédé cognitif. Les modalités de la présence des personnes dans l'espace de l'action sont également des éléments de la matérialité des pratiques.

Troisièmement, dans la version soviétique du dispatching, les procédés de communication par téléphone ou par signaux occupent une place essentielle. Ce sont les techniques qui assurent la coordination entre le poste du dispatcher et les ateliers, plus précisément qui assurent l'identité entre les tableaux d'engagement dans le premier et dans les seconds. Il s'agit d'information dans les deux sens : dans l'un vers le dispatcher et dans l'autre vers les responsables des ateliers et des transports internes divers et vers les opérateurs eux-mêmes. La littérature soviétique insiste sur le fait que le dispatching ne doit pas être confondu avec une simple gestion des aléas par le téléphone, travers dans lequel seraient tombées de nombreuses entreprises. La communication doit être assurée par des réseaux spéciaux, des commutateurs grâce auxquels

45. Voir par exemple les chapitres respectifs des classiques Dexter S. Kimball, Principles of industrial organization, New York, McGraw-Hill, 4e éd. 1933 (1 re éd. 1913), 460 p. et William B. Cornell, Organization and management in industry and business, New York, The Ronald Press, 1947 (1re éd. 1928), 819 p.

46. G. Cihockij, op. cit., p. 66 (la parenthèse est d'Y.C.).

47. Un cas de bureaucratisme caractérisé est, dans une usine, celui des techniciens chargés de coordonner la production et qui se contentent d'enregistrer et de répercuter les informations sur les pannes ou les ruptures d'approvisionnement sans sortir de leur bureau ou, dans les administrations centrales, celui des fonctionnaires qui se contentent de recevoir les agents en mission sans se déplacer sur le terrain. 
les signaux adaptés sont transportés. On a sans doute là un usage direct des procédés de dispatching en cours dans les chemins de fer ou dans la distribution électrique et transféré à l'industrie manufacturière. D'une part on ne trouve pas cette insistance sur les réseaux d'information par signaux dans la littérature occidentale généraliste. D'autre part on ne saurait manquer de remarquer qu'il y a là comme un écho de l'idéal télégraphique : un système approprié de signaux qui assureraient par leur autorité propre la viabilité, le « fonctionnement » de l'ensemble. La perspective de l'administration automatique est ainsi toujours présente, mais comme un horizon.

L'appareillage de la signalisation paraît encore largement une affaire d'instituts et de laboratoires de recherche. Cihockij note que «l'appareillage pour la gestion automatique et pour l'administration à distance se trouve au stade de la recherche scientifique $»^{48}$. L'organisme qui pilote ces recherches, comme plus généralement celles sur le dispatching, dépend du NKTP. C'est le CIO (Cental'nyj Institut Organizacii), l'Institut central d'organisation de la production et de gestion scientifique, créé en 1931 en partie pour remplacer l'institut de Rozmirovič, tout en reprenant en partie le même personnel.

Autre caractéristique enfin, les opérateurs humains ne sont plus de simples régulateurs de processus matériels. L'intensification technique du côté de l'appareillage de fiches et de signaux leur fait gagner des « qualités » (kačestva) données pour très complexes. Une de ces qualités majeures est la rapidité en général et la rapidité de réaction à l'imprévu en particulier. Elle doit être accompagnée de facultés de concentration, de maîtrise de soi, de résistance à la fatigue, etc. Ces «qualités psychologiques » sont étudiées dans un laboratoire de psychotechnique (dépendant de l'Institut d'économie et de sécurité du travail). Leur énoncé les fait d'ailleurs paraitre comme tout à fait formatées par la psychotechnique, par leur mesurabilité même par des appareils et des méthodes psychotechniques ${ }^{49}$. Dans cette version d'une gestion automatique en devenir, on voit que « l'élément humain » du système n'échappe lui-même à l'automatisme de la psychologie clinique que pour retomber dans les « aptitudes » mesurées de la psychologie expérimentale !

Le dispatching expose une intensification technique de la pratique administrative qui ne va pas sans une intensification psychologique, dans le cadre d'une intensification du commandement vertical. Nous ne sommes ni dans une « diffusion » ni dans une «hybridation », mais dans une composition complexe émergente où les traits d'origine des références sont presques perdus.

\section{L'idéal mis en échec}

Le mouvement pour le dispatching s'avère rapidement un échec aux deux niveaux. En bas, de nombreuses entreprises traînent manifestement les pieds devant cette nouvelle lubie du centre. Les schémas standard de dispatching sont imposés d'en

48. G. Cihockij, op. cit., p. 56.

49. Ibid., p. 57 
haut, identiques partout, quel que soit le type de production en question, comme l'avaient été les schémas d'organisation fonctionnelle dans les années 1920. La grande usine Putilov de Leningrad, devenue usine Kirov en décembre 1934 à la mort du secrétaire du parti de Leningrad qui portait ce nom, est la bonne dernière de la région et semble en être encore, au bout de deux ans, à l'élaboration de projets pour la mise en place du dispatching ${ }^{50}$. Il est vrai que le $\mathrm{CIO}$ a tardé à publier ses « règlements » pour la mise en dispatching des entreprises de mécanique. Il est vrai aussi que le dispatching, plus encore dans les usines de mécanique aux pratiques discrètes que dans les installations de gestion de flux comme l'électricité ou les chemins de fer, suppose la réinterprétation de toutes les activités pour les passer aux normes de l'outil de gestion et, par suite, la revisite de leurs pratiques par tous les acteurs. Malgré des séjours de plusieurs mois au sein des usines, les dispatchers des directions centrales reviennent le plus souvent amers et découragés ${ }^{51}$. Le dispatching s'installe donc avec difficulté au niveau de l'entreprise : il rajoute de surcrôit une instance qui entre en conflit avec toutes les autres puissances, et en particulier celle des chefs d'atelier appuyés sur leur autorité acquise par le fait que la production effective repose sur eux.

Comme tous les «systèmes » dans l'ambiance soviétique, celui-ci se décompose sans pouvoir surmonter le chaos qu'il est destiné à combattre. La version soviétique du dispatching paraît conférer aux dispatchers des responsabilités épuisantes. Les dispatchers deviennent souvent des tolkači, ces agents chargés de trouver à n'importe quel prix les produits manquants, quand ce ne sont pas des tolkači qui sont nommés dispatchers ${ }^{52}$. Le dispatching se transforme alors en un simple procédé d'obtention des pièces déficitaires, tout à fait comme le stakhanovisme est rapidement devenu un système de primes ordinaire ${ }^{33}$.

Au niveau du ministère, les autorités supérieures des directions paraissent très réticentes à écouter les récits de désordre et de pagaille (bezobrazija) que rapportent

50. G. Cihockij, op. cit., p. 65. I. I. Rybakov, « Dispečerizacija i sistema snabženija na zavode "Krasnyj Putilovec" » (Dispatching et système d'approvisionnement à l'usine Putilov rouge), Bjuleten' CIO, 6, 1934 ; CIO, Proekt dispečerizacii Kirovskogo Zavoda (b. 'Krasnyj Putilovec'), Traktornyj Ceh MHI (Projet de dispatching pour l'usine Kirov, anciennement Putilov rouge. Atelier des tracteurs - Première mécanique), s.l., Izd. CIO, 1935 ; Orgametall, Metodika proektirovanija $i$ vnedrenija disperčirovanija. Opyt Kirovskogo Zavoda v Leningrade (Méthodologie du projet et introduction du dispatching. L'expérience de l'usine Kirov à Leningrad), Moscou, Orgametall, 1935, 35 p.

51. Rapport cité de Nikolaev.

52. David Granick, The management of the industrial firm in the USSR. A study in Soviet economic planning, New York, Columbia University Press, 1954, p. 110-119.

53. Lewis H. Siegelbaum, Stakhanovism and the politics of productivity in the USSR : 1935 1941, Cambridge, Cambridge University Press, 1988, 326 p. ; Francesco Benvenuti, Fuoco sui sabotatori ! Stachanovismo e organizzazione industriale in URSS : 1934-1938, Rome, Valerio Levi Editore, 1988, 467 p. ; Robert Maier, Die Stachanov-Bewegung 1935-1938. Des Stachanovismus als tragendes und verschärfendes Moment der Stalinisierung der sowjetischen Gesellschaft, Stuttgart, Franz Steiner, 1990, 441 p. La dernière mise au point est R. W. Davies, Oleg Khlevnyuk, «Stakhanovism and the Soviet economy », Europe-Asia Studies, 54, 6, 2002 , p. 867-903. 
les dispatchers, et tout l'effet attendu de ce lien du centre avec les usines individuelles est perdu.

Le dispatching reste ainsi une figure très technique que prend le commandement vertical dans les entreprises, au cours des réformes qui accompagnent la Grande Terreur et qui vont toutes dans le même sens. Ces modernisations administratives dont les purges sont l'occasion ont été moins étudiées que les purges elles-mêmes. Comme l'indique David Shearer, à mesure que les pratiques du management scientifique disparaissent, des pratiques anciennes renaissent, comme le marchandage entre les ateliers et les usines ou le pouvoir de la hiérarchie directe ${ }^{54}$. Toutefois, les efforts pour installer le dispatching donnent en même temps à l'entreprise des traits modernes qui se révèleront durables. Le dispatching devient, de façon plus familière qu'à l'Ouest, une forme propre de répartition des tâches et le dispatcher un personnage installé dans les firmes et dans nombre d'institutions, comme dans le système des urgences médicales. Le dispatching est une configuration particulière des modes d'organisation soviétiques à partir de la fin des années 1930. Il est une forme d'américanisation comme une autre, c'est-à-dire un emprunt qui se situe et doit se comprendre au sein de la mise en forme de pratiques complexes sans fournir en aucune manière l'intelligibilité de celles-ci, mais seulement le nom d'une référence parmi d'autres visant à dénoter la modernité. Il ne saurait être pris non plus comme un masque qui dissimulerait un retour au passé. Il est un signe fort d'une affirmation de modernité qui ne dit rien de l'efficacité réelle des dispositifs. Il est encore une incarnation, une actualisation d'un idéal télégraphique et automatique de gouvernement proprement soviétique et, à ce titre (officieux), il n'est justement pas interrogé sur son efficacité.

Une nouvelle occurrence de ce même mouvement d'émergence de l'idéal incarné intervient dans les années 1960 et 1970 sous la forme de l'ASU (avtomatizirovannaja sistema upravlenija), système de gestion automatisé, fondé cette fois non plus sur un système de fiches de carton mais sur le système électronique de fiches qu'est, au sens propre, l'ordinateur. L'enthousiasme est infiniment plus fort que celui qui porte le dispatching dans les années 1930 : celui-ci ne pouvait pas promettre un lien de subordination automatisé pour tout l'appareil économique qu'un «système automatisé général » informatique paraît au contraire rendre réaliste au début des années 1970. Ce système doit couvrir toute l'économie de son réseau vertical depuis le sommet du Gosplan jusqu'à chaque unité économique individuelle. Les résultats sont pitoyables, inversement proportionnels aux investissements «colossaux» dont ce «système général » est l'objet à tous les niveaux de l'édifice économique ${ }^{55}$.

L'idéal d'une gestion automatique ou d'une administration transformée en technique, toujours en échec, se reproduit et survit à ses premiers tenants des années 1920 au sein de la RKI. La série se constitue ainsi : mécanisation de la comptabilité, systèmes de fiches, dispatching, automatisation informatique des systèmes de

54. D. Shearer, Industry, state..., op. cit., p. 222-224.

55. M. Beissinger, op. cit., p. 246-260 ; Slava Gerovitch, «Striving for "optimal control” : Soviet cybernetics as a "science of government" », in Miriam R. Levin, dir., Cultures of control, Amsterdam, Harwood Academic Publishers, 2000, p. 247-264. 
gestion. Le fichier (dispositif de fiches mobiles) vient se placer au centre de la rationalisation des pratiques administratives au $\mathrm{XX}^{\mathrm{e}}$ siècle, comme l'indiquaient déjà des historiennes comme JoAnne Yates et Delphine Gardey. Or cet outil administratif de plus en plus courant revêt au cours de ce siècle une signification très particulière dans la mesure où les répressions de masse qui en sont une caractéristique majeure s'appuient sur un raffinement toujours plus grand du fichage des personnes sur fichier, et non sur registre, et que l'antisémitisme en général et l'extermination des juifs d'Europe en particulier en ont fait un usage extensif ${ }^{56}$.

\section{Histoire technique de la politique et histoire politique de la technique}

Les fiches sous toutes leurs formes ne sont qu'un aspect de la matérialité des pratiques administratives. Le téléphone en est un autre qui, à ma connaissance, n'a pas fait l'objet de recherches historiques spécifiques sur l'espace soviétique, à l'inverse de l'électricité ${ }^{57}$. Cela pourrait être intéressant à de multiples titres dont certains seulement sont évoqués ici, et trop rapidement. Les économistes n'ont pas manqué de noter très tôt l'économie que le téléphone apporte dans le gouvernement des entreprises en permettant l'accroissement de leur taille: "Les changements comme le téléphone ou le télégraphe, lesquels réduisent les coûts d'organisation spatiale, tendent à accroître la taille de la firme, de même que tout changement qui améliore la technique de gestion », écrit en 1937 l'économiste Ronald Coase ${ }^{58}$. La réflexion peut clairement valoir pour le gouvernement des États et importe d'autant plus pour l'URSS qui est non seulement un immense pays, mais un pays à l'État faible à l'origine, entre autres dans sa capacité de couverture territoriale.

Mais on pourrait s'intéresser aussi à la relation entre le type de régime politique et le téléphone comme instrument de communication à distance. Tout d'abord, les chercheurs le désignent comme un moyen de communication informel, non pas tant parce qu'il ne laisse pas naturellement de trace mais, pragmatiquement, parce qu'il ne sert pas pour scander les pratiques de marques réglementaires ou légales comme une attestation écrite ou une signature. Le télégraphe, qui doit être encodé par un opérateur, penche vers le formel. Les premiers usages du téléphone se déploient

56. Sans aller jusqu'à l'usage de machines mécanographiques, comme l'a montré la controverse autour du livre d'Edwin Black, IBM et l'holocauste, Paris, R. Laffont, 2001, 595 p. (trad. de IBM and the Holocaust, New York, Crown Books, 2001) : voir par exemple Michael Allen, "Stranger than science fiction. Edwin Black, IBM, and the Holocaust », Technology and Culture, 43, 1, janvier 2002, p. 150-154.

57. Par exemple, Jonathan Coopersmith, The electrification of Russia, 1880 to 1926 : Power plants, politics and people, Ithaca-Londres, Cornell University Press, 1992, 274 p. [Je ne prends connaissance qu'au moment de la relecture des épreuves de l'existence de l'ouvrage de Vladimir V. Pavlov, Pravitel'stvennaja elektrosvjaz'v istorii Rossii. Cast'1 : 1971-1945 (Les télécommunications électriques gouvernementales dans l'histoire de la Russie. $1^{\text {re }}$ partie : 1917-1945), Moscou, Nauka, 2001, 358 p.]

58. Ronald H. Coase, « La nature de la firme », Revue française d'Économie, III, 1, hiver 1987, p. 145 (éd. américaine en 1937). 
dans les affaires. Ils sont de type managérial ${ }^{59}$. Ensuite, il n'est pas un ouvrage sur l'histoire du téléphone qui n'insiste sur le fait que le téléphone se présente d'emblée comme un équipement du commandement, comme un outil hiérarchique. Les usages relationnels et privés n'arrivent qu'ensuite ${ }^{60}$. La chose n'est plus à montrer. L'histoire féministe attire de son côté l'attention sur cet autre fait que le téléphone, dans sa réalité pratique comme dans ses représentations, met le plus souvent en scène le patron ou le chef - masculin - et la secrétaire ou la « demoiselle du téléphone »- féminine ${ }^{61}$. En troisième lieu et contrairement à ce que disent certains chercheurs, le téléphone n'est donc pas naturellement «égalitaire», pas plus qu'internet n'est aujourd'hui démocratique en soi. Ce n'est pas la liaison isolée entre un combiné et un autre qui compte dans la portée sociale du téléphone mais le réseau installé. L'objet technique est le réseau et non un de ses éléments pris séparément. Le réseau n'est pas ici un « quasi-objet ». Il a une pleine matérialité objectale qui ne se comprend que comme élément d'un système technique organisé, mis en place, relevant d'une organisation, d'une administration, d'une gestion et d'une politique. La fin est dans le moyen et le moyen ici est un système technique dans son ensemble ${ }^{62}$.

Le téléphone se définit longtemps dans une concurrence avec le télégraphe. Or c'est une bataille politique qui préside aux États-Unis à l'invention du téléphone. Gardiner Hubbard, l'homme d'affaires qui chapeaute dès avant les années 1880 les travaux de Graham Bell sur le téléphone, entend combattre le monopole de la Western Union sur le télégraphe. Il cherche à promouvoir la démocratie, à protéger les citoyens contre un monopole sur la circulation des informations et sur leur contrôle. Le téléphone se profile comme un outil pour échapper à ce contrôle corporatif (sur les prix de marché, sur les informations de bourse, etc.) sans tomber dans un contrôle par l'État (quoique les opérateurs reviendront dans le téléphone sous forme de «demoiselles », tandis qu'on pensait les avoir chassés en délogeant le

59. Voir J. Yates, op. cit.

60. Catherine Bertho, Télégraphes et téléphones de Valmy au microprocesseur, Paris, Le Livre de poche, 1981, 538 p. et id., dir., Histoire des télécommunications en France, Toulouse, Erès, 1984, 267 p. ; Claude F. Fischer, America calling : A social history of the telephone to 1940, Berkeley, The University of California Press, 1992, 424 p. Guy Thuillier, La vie quotidienne dans les ministères au XIXe siècle, Paris, Hachette, 1976, p. 195-196: «J'ai été témoin de l'introduction au ministère de cet instrument diabolique, note Claudel. Les vieux expéditionnaires ne purent jamais s'habituer à cette voix maléfique qui leur apportait on ne sait d'où les commandements et les consignes de l'inconnu. Le père $\mathrm{D}$., une fois que la plaque vibrante lui apportait une question du ministre - M. le ministre lui-même ! - lâcha instantanément le cornet pour endosser sa redingote. »

61. Delphine Gardey, « Humains et objets en action : Essai sur la réification de la domination masculine », in Delphine Gardey et Danièle Chabaud-Rychter, dir., L'engendrement des choses. Des hommes, des femmes et des techniques, Paris, Éditions des archives contemporaines, 2002, p. 239-267.

62. Sur la notion de système technique, voir Thomas P. Hughes, « The evolution of large technological systems », in Wiebe E. Bijker, Thomas P. Hughes et Trevor Pinch, dirs, The social construction of technological systems, Cambridge (Mass.), MIT Press, 1987, p. 51-82 et id., «L'histoire comme systèmes en évolution », Annales. Histoire, sciences sociales, 53, 4-5, juil.oct. 1998, p. 839-857. 
télégraphe). Hubbard veut une technique qui supporte aussi bien les messages privés que les messages d'affaires ${ }^{63}$. Le téléphone (comme réseau) est un objet qui a une politique, mais il est susceptible d'avoir diverses politiques selon la manière dont il est conçu, installé et utilisé64. Il est un de ces objets qui permettent d'envisager tout autant une histoire politique de la technique qu'une histoire technique de la politique.

Quant à l'histoire soviétique, la question se pose de savoir si le téléphone a contribué à la dictature stalinienne (on pourrait plus généralement poser la question de savoir s'il a contribué aux dictatures du Xxe siècle). En d'autres termes, la compréhension du stalinisme peut-elle se priver du téléphone ? Il faudrait, pour répondre à cette question, une large enquête qui soit orientée sur les pratiques. Trop souvent, les textes historiens relatifs au téléphone en restent aux représentations élaborées à partir des publicités et des articles de promotion, sans entrer dans les pratiques effectives au sein des organismes divers. L'étude des premières ne saurait aucunement tenir lieu de l'étude des secondes. Je ne peux cependant pas aller plus loin que la fourniture de quelques maigres flashs illustratifs de ce que pourrait être une telle histoire pragmatique et politique commune du téléphone et du stalinisme.

\section{Le téléphone et l'arbitraire}

Tout d'abord, les histoires du téléphone traitent peu de l'écoute des conversations. Or celle-ci vient très vite. Guy Thuillier relève qu'en France, dans les bureaux de poste, « la pratique des écoutes téléphoniques est fort ancienne : avant 1914, dans tous les bureaux de Paris, "il y a une liste de 30 à 40 personnes qui sont écoutées" ». Dans l'administration, les hauts fonctionnaires préfèrent faire porter les messages autant par crainte du cabinet noir que des écoutes ${ }^{65}$. Stalin est ici encore une fois un maître, un maître encore une fois d'une manière toute particulière en ceci qu'il n'est pas un inventeur, mais un grand développeur, comme nous le verrons derechef bientôt.

Et c'est encore Bažanov qui fournit sur cet épisode le seul récit disponible, ce qu'il restera sans doute puisqu'il traite là du dernier degré du secret. En 1922, Lenin fait installer au Kremlin un «tourniquet » (vertuška), c'est-à-dire un central automatique fermé, limité aux quelques dizaines d'appareils des principaux dirigeants soviétiques, membres du Politbjuro et secrétaires du Comité central : il s'agit

63. W. Bernard Carlson, «The telephone as political instrument : Gardiner Hubbard and the formation of the middle class in America, 1875-1880», in Michael Thad Allen, Gabrielle Hecht, dir., Technologies of power : essays in honor of Thomas Parke Hughes and Agatha Chipley Hughes, Cambridge (Mass.), MIT Press, 2001, p. 25-55.

64. Langdon Winner, La baleine et le réacteur. À la recherche des limites au temps de la haute technologie, Paris, Descartes \& Cie, 2002 (le chapitre «Les artefacts font-ils de la politique ? », éd. originale américaine « Do artifacts have politics ? », Daedalus, 109, 1980, p. 121-136).

65. Guy Thuillier, La bureaucratie en France auX XIXe et XXe siècles, Paris, Économica, 1987, p. 703, qui cite Benoît Laurent, Les services postaux en 1913, thèse de droit, Lyon, 1913, p. 180. 
d'éviter que leurs conversations ultra secrètes passent par les demoiselles du téléphone (soit de nouveau s'affranchir de l'interférence humaine dans une activité de direction comme dans le système de fiches automatique). Stalin, qui vient d'être nommé secrétaire général, se charge de l'exécution. Or le poste de contrôle qui accompagne toute installation de ce type est judicieusement placé dans un tiroir de son bureau et Stalin a dès lors accès à toutes les conversations de tous ceux qui lui importent. Le communiste tchèque spécialiste des centraux automatiques qui a installé le tourniquet est exécuté par la GPU. Selon Bažanov, trois personnes dont lui-même sont, en sus du secrétaire général, au courant de cette pratique qui offre à Stalin un atout incommensurable dans sa lutte pour le pouvoir ${ }^{66}$. Le secret recherché par Lenin pour un happy few est poussé à sa limite extrême au profit d'Un seul, par une simple ruse littéralement bureau-cratique (et un assassinat). La mètis, ou encore l'astuce technique, contribue puissamment à la victoire politique ${ }^{67}$.

Le système d'écoutes est un usage des pouvoirs du téléphone au second degré. En ce qui concerne Stalin, le premier degré de cet usage du téléphone, l'intervention directe, est largement documenté. D'une façon générale, le téléphone se présente comme un remarquable instrument de contrôle par les supérieurs hiérarchiques. Il s'est ainsi imposé comme outil de la conduite de la guerre à la faveur du premier conflit mondial et l'on n'a pas tardé à s'apercevoir dans l'armée que son utilisation favorisait la propagation d'une « téléphonite » aiguë. Celle-ci était bien entendu d'abord le fait des instances supérieures. Il s'est en effet très vite manifesté « une tendance des Grands Quartiers Généraux à interférer dans le plus petit détail simplement parce qu'on pouvait le faire très facilement», comme l'indique un historien militaire ${ }^{68}$. Instrument du commandement à distance, le téléphone permet aux chefs lointains de traverser l'espace instantanément pour s'informer ou pour intervenir.

Il faudrait rassembler en Union soviétique les récits dispersés des interventions directes des hiérarchies et en particulier de Stalin par ce moyen auprès des personnes et auprès des organismes les plus variés.

Bien souvent, ces intrusions sont menées de façon peu régulière, sans souci des niveaux hiérarchiques ni des limites organisationnelles. Le téléphone se fait alors non pas l'outil de l'arbitraire mais sa manifestation même. En d'autres termes, ce n'est pas parce qu'il y a un pouvoir arbitraire qu'il y a cet usage du téléphone mais c'est du fait de cet usage que le pouvoir est arbitraire. Un seul exemple en ce qui concerne Stalin, le coup de téléphone adressé le 18 avril 1930 à un Bulgakov désespéré. Après s'être assuré que l'écrivain voulait rester en Union soviétique, Stalin l'invite à adresser une nouvelle demande d'embauche au Théâtre d'art de Stanislavskij dont il avait reçu auparavant une réponse négative : «Faites donc une

66. B. Bajanov, op. cit., p. 54-57.

67. Marcel Détienne et Jean-Pierre Vernant, Les ruses de l'intelligence ou la mètis des Grecs, Paris, Flammarion, 1978, 316 p. (1éd. 1974).

68. Martin L. van Creveld, Command in war, Cambridge (Mass.), Harvard University Press, 1985, p. 169. 
demande. Je pense qu'ils accepteront $»^{69}$. Ce qui fut fait. Le téléphone est l'arbitraire du dictateur de façon plus intense que pour n'importe quel chef. Il peut être aussi l'institutionnalisation de l'arbitraire, comme plus tard, aux temps brejnéviens, ce que la sagesse populaire appelle le «droit téléphonique » (telefonoe pravo), par lequel les instances du parti dictent les sentences aux organes judiciaires en une pratique devenue ordinaire.

Sous un autre angle, toujours au chapitre de l'arbitraire "performé » par le téléphone (comme on dit en pragmatique), les historiens du gouvernement soviétique montrent comment son usage croît sous Stalin pour consulter les dirigeants plutôt que les réunir physiquement ou leur soumettre des documents, et cela autant pour le Politbjuro que pour le Conseil des commissaires du peuple ${ }^{70}$.

\section{Intervention téléphonique et prolifération bureaucratique}

Une enquête historique pourrait dire comment et jusqu'à quel point la pratique de l'intervention téléphonique s'est répandue. Dans le massif administratif soviétique, elle est tout à fait typique d'un régime où ce qui est du parti et ce qui est de l'appareil gouvernemental sont difficilement partageables. Le téléphone permet de passer sans trace - de façon, dirions-nous improprement aujourd'hui, «immatérielle »les frontières affichées qui séparent l'un et l'autre, sans s'arrêter à des opérations lourdement matérielles et formelles. Le téléphone est de ces objets qui démontrent la vanité de vouloir séparer les pratiques administratives (c'est-à-dire, pour ce pays, les activités politiques) qui ont leur siège dans l'une ou l'autre institution de l'État et du parti.

Du fait de cette facilité qu'il offre à franchir les frontières formelles, le téléphone est en même temps la hantise des pouvoirs et des hiérarchies. Question de base : comment en éviter un usage résistanciel ? En restreignant la distribution, assurément. Réglementation, écoutes, tarifs. Je ne traite pas ici la question pour l'URSS. En revanche, j'aimerais évoquer un point inaperçu qui est une autre hantise, celle des hiérarchies intermédiaires devant la propension de leurs supérieurs à les contourner par le téléphone. Du point de vue de la matérialité, l'écrit est infiniment plus lent et il laisse plus de traces. L'écrit perd en efficacité ce qu'il gagne en limitant les contournements hiérarchiques. Le téléphone se présente paradoxalement comme une technique qui combat la relation bureaucratique mais qui renforce en fait la centralisation. Ordžonikidze, commissaire du peuple à l'Industrie lourde de 1932 à 1937, principal ministre économique, était un praticien intensif du téléphone à longue distance physique et hiérarchique. Le roman de Bek, La nouvelle affecta-

69. Paul Kalinine, présentation de Mikhail Boulgakov, La fuite. Les journées des Tourbine, Paris, Robert Laffont, 1971, p. 38. Voir le commentaire de Michel Heller, La machine et les rouages, Paris, Gallimard, 1994, p. 92.

70. Oleg Khlevniouk, Le cercle du Kremlin. Staline et le Bureau politique dans les années 30 : les jeux du pouvoir, Paris, Seuil, 1996, p. 78, 123, 138, 178, 266, 318 ; Derek Watson, Molotov and Soviet government. Sovnarkom, 1930-41, Londres-New York, Macmillan, 1997, p. 74. 
tion, explore l'action d'un ministre de l'Industrie d'après-guerre dont le téléphone est aussi le principal instrument de commandement et d'intervention ${ }^{71}$.

Apparemment, ce n'est pas propre au cas soviétique. L'inquiétude des hiérarchies intermédiaires paraît la même ici et là. Ainsi deux épisodes presque semblables dans deux usines, l'une soviétique, l'autre française, l'usine Kirov de Leningrad et Peugeot. En 1939, le directeur de l'usine Kirov (jusqu'en décembre 1934 usine Putilov), demande à tous ses chefs de département de soumettre à sa signature les réponses qu'ils adressent aux demandes de renseignement du commissaire du peuple ou d'un de ses adjoints. Ces demandes qui viennent du sommet sont adressées directement à ses subordonnés et le plus souvent par téléphone (quoique parfois aussi par lettre ou télégramme). Le directeur doit intervenir spécialement pour être informé 72 . De façon identique, en 1937, le directeur des fabrications de Peugeot fait remarquer à son directeur général que celui-ci ne le prévient jamais lorsqu'il adresse des demandes d'information ou des instructions à ses subordonnés par téléphone, alors que toutes les demandes et les instructions passaient par lui lorsqu'elles étaient écrites. Il dénonce l'usage du téléphone comme un danger pour l'unité de direction, pour «l'unité d'autorité », comme on disait au XIX' siècle, car ses cadres sont susceptibles d'échapper à sa « surveillance » et à son « contrôle » ${ }^{73}$.

Il existe donc un usage constant du téléphone en infraction au principe hiérarchique qui veut que toute communication passe par tous les niveaux. Cet usage, et je parle bien de cet usage précis, non pas du téléphone en général, favorise ainsi la dissolution des autorités intermédiaires et la fragilisation des administrations décentralisées.

Autre effet du téléphone : l'assignation des cadres à la place. Les cadres supérieurs des entreprises sont chroniquement sur-occupés, en particulier par des réunions des instances les plus diverses hors de leur espace de direction (parti local, administration de branches, police politique, Moscou et ses merveilles administratives), sans parler des réunions internes ${ }^{74}$. En 1930, le conseil de l'économie pour la région de Leningrad émet un ordre (prikaz) spécial pour obliger les directeurs d'usine à rester dans leurs locaux et à s'entendre par le téléphone avec les instances extérieures :

En vue d'assurer une direction effective (dejstvitel'noe rukovodstvo) des entreprises, j'interdis à tous les directeurs et à leurs adjoints de s'absenter de l'entre-

71. Kendall E. Bailes, Technology and society under Lenin and Stalin (1917-1941), Princeton, Princeton University Press, 1978, p. 283, qui cite le fort riche I choose freedom, de Viktor Kravchenko (J'ai choisi la liberté. La vie publique et privée d'un haut fonctionnaire soviétique, Paris, Self, 1947, 638 p.). A. Bek, La nouvelle affectation, Paris, Messidor, 1988, 298 p. (Novoe naznačenie, Moscou, Izd. Knižnaja Palatka, 1987, 214 p.)

72. GASP (Archives d'État de Saint-Pétersbourg), 1788/31/19/189, note de service de I. Zal'cman, 29 octobre 1939

73. Archives Mattern, Mattern à J.-P. Peugeot, 15 mai 1937, manuscrit.

74. Iosif M. Burdianskij, Osnovy racionalizacii proizvodstva (Fondements de la rationalisation de la production), Moscou, OGIZ 'Moskovskij Rabočij', 2 éd. rev. \& augm. 1931, p. 324. Voir aussi Hiroaki Kuromiya, Stalin's industrial revolution : Politics and workers : 1928-1932, Cambridge, Cambridge University Press, 1988, p. 75. 
prise sans nécessité absolue dans le cours de la journée de travail. La sortie de l'entreprise sur convocation de diverses organisations et institutions n'est autorisée que dans des cas exceptionnels et en raison d'une impossibilité totale de s'entendre avec les institutions et organisations en question par téléphone ${ }^{75}$.

La sortie du bureau est en revanche encouragée pour des visites d'inspection sur le terrain. Le téléphone fait son office d'instrument de gouvernement à distance. Il favorise la sédentarisation des dirigeants et des administrateurs... et, de ce fait, exactement ce qui est dénoncé en Union soviétique comme bureaucratisme. Le paradoxe se prolonge si l'on songe que, dans le cas indiqué, il est un instrument disciplinaire pour le management intermédiaire de l'industrie tandis que, dans le cas personnel de Stalin, il aide un dictateur, qui répugne à en sortir, à rester dans son bureau du Kremlin ou ses résidences de vacances, ce chef fournissant à tous les cadres l'exemple de ce qui leur vaut sa sollicitude répressive.

Un autre aspect, complémentaire, semble devoir être documenté davantage. Il s'agit de l'allocation du téléphone à ces hiérarchies intermédiaires, à la fois pour les usages de direction, de contrôle et de discipline indiqués et comme compensation à la propension irrépressible des supérieurs à les contourner par ce moyen même. Pour une époque bien postérieure et en parlant du sommet, Tamara Kondratieva relève qu'une place sur le tourniquet, héritier de celui de Lenin, est le privilège suprême pour les cadres du Kremlin. La dotation en téléphone représente certainement un signe de l'installation hiérarchique aussi fort que l'allocation de privilèges de consommation ${ }^{76}$. Il est une marque de pouvoir, comme le bip des cadres que met lumineusement en scène l'anthropologue Véronique Moulinié dans une entreprise française ${ }^{77}$. Le téléphone en Union soviétique n'est toutefois ni seulement un signe, ni seulement un privilège hiérarchique. L'usage de la filmographie soviétique comme source montre la valeur extraordinaire accordée au téléphone, qui est celle même de la liaison avec le centre ${ }^{78}$. Le téléphone est un des principaux acteurs matériels des films soviétiques de l'entre-deux-guerres, en concurrence avec le tracteur, la locomotive et l'usine... Il est la marque propre à l'Union soviétique, et peut-être aux pays communistes, d'une relation privilégiée avec ce qui importe, le centre.

Sur un autre plan encore, il n'est pas seulement un signe. Il fait effectivement quelque chose dont l'aspect principal est de créer à distance (distance spatiale,

75. Dans l'exemplaire destiné à Putilov, la signature des cadres de l'usine concernés est portée en haut et à gauche du document comme marque de sa consultation. GASP, 1788/23/137/120, 17 septembre 1930

76. Tamara Kondratieva, Gouverner et nourrir. Du pouvoir en Russie (XVIe-XXe siècles), Paris, Les Belles Lettres, 2002, p. 188-189 ; voir aussi Elena A. Osokina, Ierarhija potreblenija ( $L a$ hiérarchie de la consommation), Moscou, Izd-vo MGU, 1993, 144 p.

77. Véronique Moulinié, «La passion hiérarchique. Une ethnographie du pouvoir en usine », Terrain, 21, octobre 1993, p. 129-142.

78. Par exemple, Zemlja (La terre), de Dovženko (1930), Lenin v 1918 godu (Lenin en 1918), de Mihail Romm (1938), avec le télégraphe comme acteur central, Člen pravitel'stva (Membre du gouvernement), d'Aleksandr Zarhi et Iosif Hejfic (1940) ou Kommunist (Le communiste), de Rajzman, 1957. 
sociale ou hiérarchique) un effet de présence. La mise en présence téléphonique a, dans les situations, non pas tant une signification particulière qui s'ajoute au sens déclaré porté par les messages : elle est une part de la composition du sens de ces messages qui lui restent indexés. C'est à ces multiples titres que le téléphone fait émerger, performe, l'autorité.

Pour résumer, utilisé pour obvier aux rigidités bureaucratiques, il passe les bornes administratives et a ce premier effet de provoquer une prolifération de papier pour mieux contrôler ces franchissements et ce second de renforcer la centralisation et la non-mobilité. La parole téléphonique engendre de l'écrit et de la stratification sociale - les techniques procèdent du social et en créent. Le « large réseau d'appareils » dont doit, selon Stalin, s'entourer le parti perd de nouveau sa consistance métaphorique pour prendre cette fois substance téléphonique (tandis que le télégramme, chiffré ou non, lâche de plus en plus sa position dominante). Le téléphone réalise, si l'on se place du point de vue du seul Stalin, l'idéal télégraphique. Mais la multiplication des pratiques bureaucratiques et des grappes sociales attachées au téléphone que cela provoque échappe au contrôle de Stalin et, avec la centralisation renforcée, contribue à conformer la société soviétique.

Le téléphone consolide les systèmes centralisés et, plus encore, il est un outil privilégié des dictatures du XXe siècle ${ }^{79}$. Il a bien une action sur l'espace du commandement et du contrôle. Mais parmi tous les possibles, le fait que le téléphone ait actualisé sa propre propension à assister l'arbitraire et à accroître l'étendue de son emprise, sinon son efficacité, est bien un événement du XXe siècle. Et ceci, contre un téléphone relationnel ouvert à un usage résistanciel et égalitaire d'où serait susceptible de surgir la politique, comme des cuisines de l'URSS tardive.

\section{La matérialité policière de l'articulation parti-gouvernement}

Les formalités administratives ont toujours revêtu en URSS des formes lourdement matérielles. Une grande part d'entre elles relève à coup sûr de l'héritage de l'ancienne administration et les pratiques administratives sont sujettes aussi à une continuité qu'Octobre ne brise pas de façon marquée. Toutefois, la problématique de la continuité, qui n'est pas développée ici, ne doit pas masquer les formulations en situation : celles-ci opèrent le tri entre ce qui est hérité, ce qui est reproduit ou non, même si tout ne relève pas de choix entièrement délibérés. Ce qui tient à la continuité ne peut être compris que dans l'analyse de ce qui est convoqué et de ce qui est abandonné dans les situations. Ce qui n'est pas retenu est en général ignoré

79. Le communisme fait un usage systématique des moyens modernes de communication et les liaisons internationales du Komintern sont réputées les meilleures de leur temps, faisant toutefois surtout usage de la radio. Voir l'un de leurs principaux responsables évoquant « les excellents moyens de liaison (télégraphes, téléphones, radio) qui existent à l'étranger », Ossip Piatnitski, Le travail d'organisation dans les partis communistes des pays capitalistes, Paris, Bureau d'Éditions, s.d. [1928], 112 p. ; Branko Lazitch, « La formation de la section des liaisons internationales du Komintern (OMS), 1921-1923», Communisme, 4, 1983, p. 65-80 ; Natalia S. Lebedeva, Le service de liaison du Komintern entre 1939 et 1941, à paraître en 2003. 
des continuistes qui mettent surtout l'accent sur les permanences et les « résurgences ». Le sens se formule au présent. Lorsque Lenin demande en 1922 à un dirigeant de la RKI de « se procurer de la littérature allemande et américaine [et de] réunir tout ce qui a quelque valeur, surtout en ce qui concerne la normalisation du travail de bureau (règles pour la circulation des papiers, formes ; contrôle ; copies à la machine ; questions et réponses, etc.) », ce qui importe est l'insistance à introduire des règles étroites dans tout le travail administratif d'un parti au pouvoir et d'un État fragile. Il existe un fort souci au sommet pour les formes administratives et leur matérialité ${ }^{80}$. Les prescriptions quant à la circulation et à la conservation des papiers et de la correspondance secrète occupent des volumes ${ }^{81}$.

Or la continuité de l'espace de prescription entre le parti et l'appareil gouvernemental (qui incorpore l'économie), performée, entre autres, par une certaine pratique téléphonique, n'est pas de droit. Le parti dirige (de façon constitutionnelle seulement depuis la Constitution de 1936) tandis que les soviets ont le pouvoir. Ce qui a toutes les caractéristiques et les effets perturbateurs d'une double contrainte aux injonctions contradictoires est une articulation étroitement régie par le secret et inscrite avec soin dans des pratiques très matérielles, sacralisées et judiciarisées. La formalité est pesamment matérielle. Le présent paragraphe reprend des choses connues, simplement replacées sous l'éclairage de la matérialité. J'emprunte beaucoup à l'article d'Irina Pavlova sur « le mécanisme du pouvoir politique en URSS dans les années 1920-1930 »82.

Les pratiques ordinaires du secret spécifiques à l'histoire du pouvoir soviétique occupent beaucoup de personnel et de temps. Elles supposent un savoir-faire particulier, mais aussi la disposition de lieux adaptés, de bâtiments de bureau aménagés de façon idoine, de matériel spécial comme de la cire et des cachets. Elles réclament des coffres-forts avec leur pièce réservée, le respect d'interdictions de contact et de déplacement de la part des porteurs du secret et bien d'autres mesures encore. Elles n'organisent pas seulement la vie de ceux qui en ont la responsabilité directe mais celle de tous les citoyens. Elles sont en effet connues de tous et elles supposent de la part de chacun des représentations particulières de l'espace et des personnes. Ces pratiques sont indétachables du pouvoir soviétique dans son exercice quotidien et chacun y participe à un titre ou à un autre.

La période décisive pour l'installation du secret spécifique au pouvoir soviétique est la fin de 1922 et le début de 1923, dans les premiers mois des fonctions de Stalin comme secrétaire général après le début de la maladie de Lenin. Le futur

80. V. Lénine, Euvres, t. 36,p. 594 (lettre citée).

81. Voir, sur les procès-verbaux du Bureau politique et du Comité central, O. Khlevniouk, op. cit., annexe IV,p. 317-323.

82. I. Pavlova, « Mehanizm političeskoj vlasti. SSSR 1920-1930 g. », Voprosi istorii, 11-12, 1998 , p. 49-66. Dans la littérature abondante sur les « mécanismes » du pouvoir ou de la décision, c'est l'un des rares qui s'intéresse de près au mécanisme au sens propre, c'est-à-dire à la matérialité des choses et à la manière dont des personnes inventent et pratiquent ces mécanismes ici presque non métaphoriques, faisant en fin de compte du pouvoir décrit tout autre chose qu'une mécanique. 
dictateur parachève l'organisation matérielle du caractère «conspirateur » que le parti doit conserver. Le document fondamental est l'instruction sur la conservation et la circulation des documents secrets de novembre 1922, dont l'effet se prolonge jusqu'à la fin des années 1980. Les mesures qui concernent la matérialité du traitement des documents sont les suivantes. La décision du parti est transmise sous enveloppe scellée à la section secrète de l'organisme gouvernemental situé au même niveau que l'instance du parti qui a émis la directive. La transmission est opérée par une institution qui vient s'insérer en tiers : la police secrète, dotée d'un service spécialisé d'estafettes. Les trois piliers fondamentaux et associés du pouvoir soviétique sont réunis physiquement à l'occasion de cette opération : le parti, la police politique et l'administration gouvernementale. Le premier remet à la seconde ce qu'elle doit faire parvenir à la troisième pour piloter son action. Dans l'instance gouvernementale, le responsable (zavedujuščij) de la section secrète informe le chef (načal'nik) de son administration qui, se fondant sur la directive, prend une mesure en son nom propre : aucune mention de la directive du parti ne doit apparaître et aucune référence ne doit lui être faite ${ }^{83}$.

La coupure est organisée entre la décision du parti et sa mise en œuvre administrative : l'acte d'écrire la décision du parti dans les dispositions des administrations de l'État est interdit. Là est l'articulation essentielle du secret sur lequel repose le pouvoir soviétique : l'articulation est au secret comme le cadavre dans le placard. D'autres décisions précisent le système. Ainsi Stalin définit-il en personne le règlement de la correspondance la plus secrète, celle qui peut valoir 10 ans de camp et même « la mesure suprême » de punition, c'est-à-dire la mort, en cas de perte d'une enveloppe : sceller les enveloppes, ne remettre qu'en mains propres à la personne même, émarger sur le paquet, compter les documents, renvoyer le pli à l'expéditeur dans les 24 heures $^{84}$. La mesure principale est l'institution de la GPU en gardienne de l'articulation entre parti et administration gouvernementale: les clauses secrètes sont transmises exclusivement par ses coursiers. En 1926, la GPU est chargée en outre du contrôle du personnel lié au secret, c'est-à-dire des adjoints nommés pour la liaison avec elle (po OGPU) à tous niveaux de l'appareil gouvernemental. À partir de 1930, tout le territoire est couvert par le réseau des fel' $d^{\prime \prime}$ eger (coursiers rapides et motorisés) de l'OGPU. Il ressort de ces manipulations que, sur le plan de la matérialité des pratiques administratives soviétiques aussi, nous sommes confrontés à l'inexactitude de la formule courante de «parti-État»: trois termes restent associés tout au long, parti-gouvernement-police politique.

C'est Stalin qui a systématisé dans le fonctionnement ordinaire de l'État la pratique du secret déjà formalisée avant lui. Il a trouvé les formes pratiques et matérielles de la conservation du secret et institué la police politique comme garante du

83. I. Pavlova, art. cit., p. 61.

84. Dans l'appareil du Komintern aussi, on peut être condamné pour infraction à la circulation des documents secrets : Peter Huber, « L'appareil du Komintern, 1926-1935. Premier aperçu », Communisme, 40-41, 1995, p. 15. 
secret de la rencontre entre le parti et les administrations, au moment où l'articulation politique majeure se transforme en secret. On a d'ailleurs là une autre intervention sur l'espace puisqu'il s'agit de transporter un document d'un point à un autre. La police règle la circulation. Stalin a encore instauré le Secrétariat général en point de passage obligé pour toute relation secrète avec les régions sur le plan de la délibération comme de l'exécution.

Il n'y a pas là la technicité du dispatching ni le support de l'arbitraire que peut constituer le téléphone, mais une routine bureaucratique avec laquelle tout le monde est en contact à un titre ou un autre et qui porte toute la sacralité du pouvoir : une routine d'autant plus importante à conserver et à revitaliser sans cesse qu'elle est sans cesse remise en cause dans les pratiques ordinaires, celle du téléphone parmi d'autres. Cette matérialité particulière et policière de la relation imaginairement secrète entre le parti et l'État s'est maintenue jusqu'au bout.

\section{Conclusion}

Des proclamations discursives diverses, appuyées sur des métaphores aussi diverses - celles du télégraphe, de la chaîne, de l'automatisme, du travail industriel... - forment, dès les premiers pas du pouvoir bolchevik, un idéal industriel de l'État. Cet idéal passe par des actualisations répétées, indifférentes aux échecs, jamais identiques, et les métaphores se matérialisent dans des dispositifs techniques. Le dispatching est l'une de ces actualisations, introduit à tous les niveaux de l'administration industrielle à la faveur des purges de 1937-1938. Il est à comprendre comme un effort pour saisir l'administration des usines comme celle des organes industriels centraux dans la matérialité du « système de fiches » et de la transmission électrique, et ainsi résoudre les problèmes critiques de gestion apparus lors des premiers plans - attribués aux espions et aux saboteurs, c'est-à-dire aux personnes. Les systèmes de fiches sont pensés au début comme une vaste machine automatique dont les humains sont les organes régulateurs. Les humains, ensuite, sont envisagés comme traitables par la psychologie appliquée, plus précisément la psychotechnique. Ils sont traités en fait beaucoup plus par des mesures « administratives » de répression. L'ordinateur se profilant, celui-ci sera envisagé comme une machine de gestion automatique sans participation humaine. La fiche permet de développer la gestion de masse exactement comme le téléphone permet d'étendre le territoire administré.

Le téléphone, de son côté, affranchit l'arbitraire des formalités du télégraphe. Par son effet de présence, il interroge les hiérarchies en renforçant la centralisation et en provoquant une croissance de la paperasserie et un nouveau mode de stratification hiérarchique. Les formalités prennent leur revanche dans la matérialité de la transmission des documents secrets qui atteste, sous la garde de la police politique, la solidité de l'articulation fondamentale qui relie le parti à l'appareil gouvernemental. Le pouvoir est ainsi mis en circulation de diverses manières, dont celles-ci ne sont que quelques-unes. Il s'agit d'assurer une gestion efficace 
des intérêts d'un ensemble bureaucratique édifié pour que rien ne le sépare de la société. Équipé de hiérarchies variées, cet ensemble a pour politique de verrouiller toute irruption de la politique, c'est-à-dire de toute parole intempestive qui n'ait pas emprunté les chemins convenus. La société est constituée en corps de subordination traversé par toutes les formes techniques de la transmission, non sans l'aide de répressions féroces et répétées et de la criminalisation des pratiques ordinaires.

L'étude de la matérialité des pratiques dans cet espace de prescription administrative continu fait apparaître toutefois un pullulement de vie.

Ce n'est pas que l'interprétation en termes de «machine bureaucratique » ou de «la machine et les rouages» soit inexacte ${ }^{85}$. Elle est cependant à resituer historiquement comme une production intellectuelle et un programme pratique qui sert de référence récurrente, dont seraient à étudier de près les modalités d'existence, de maintien, de réitération et d'information des pratiques administratives en situation. De plus, cette interprétation en termes de machine et de rouages serait effectivement inexacte si elle empêchait l'étude des pratiques de ressaisir l'engagement des personnes là où nous serions portés, par l'effet même du langage dont nous héritons, à désigner celles-ci comme des rouages « réels», précisément comme elles se représentaient et se voyaient elles-mêmes. Le paradoxe c'est qu'à tous niveaux, des opérateurs au sommet le plus élevé, les agents de l'administration investissent leur personne pour construire un État qui puisse fonctionner comme une machine selon des règles impersonnelles et où chacun soit un rouage - et la bureaucratie vue par Max Weber et ses disciples n'est plus si impersonnelle. Autrement dit, la subjectivité est investie dans la construction bureaucratique en même temps que dans la construction de soi comme rouage, ainsi qu'un nouveau courant historiographique plein de promesses commence à le montrer ${ }^{86}$. Du coup, on n'a plus vraiment de machine. En tout cas l'usage du terme devient problématique, c'est-à-dire un problème historique à traiter. Le concept d'«appareils » selon Bourdieu, qui entend par là des champs où toutes « résistances » et « réactions » sont « annulées », ne saurait pas plus être un appui pour la pensée ${ }^{87}$. Quoique la «mécanique du pouvoir » et les « mécanismes » qui reviennent si souvent sous la plume de Foucault ne soient pas plus heureux sous ce jour, on est bien plutôt dans une forme circulatoire du pouvoir telle qu'il l'évoque lui-même : « Le pouvoir s'exerce en réseau et, sur ce réseau, non seule-

85. Voir M. Heller, op. cit.

86. Voir Jochen Hellbeck, « Speaking out: Languages of affirmation and dissent in Stalinist Russia », Kritika, 1, 1, hiver 2000, p. 71-96 et id., « Working, struggling, becoming : Stalin-era autobiographical texts », The Russian Review, 60, juillet 2001, p. 2-21 ; Brigitte Studer, Berthold Unfried, Der stalinistische Parteikader. Identitätsstiftende Praktiken und Diskurse in der Sowjetunion der Dreißiger Jahre, Cologne, Böhlau, 2001, 326 p. ; Brigitte Studer, Berthold Unfried et Irène Herrmann, dirs, Parler de soi sous Staline. La construction identitaire dans le communisme des années trente, Paris, Éditions de la Maison des sciences de l'homme, 2002, 210 p. ; Claude Pennetier, Bernard Pudal, dirs, Autobiographies, autocritiques, aveux dans le monde communiste, Paris, Belin, 2002, 367 p.

87. Pierre Bourdieu, Questions de sociologie, Paris, Minuit, 1980, p. 136. 
ment les individus circulent, mais ils sont toujours en position de subir et aussi d'exercer ce pouvoir. Ils ne sont jamais la cible inerte ou consentante du pouvoir, ils en sont toujours les relais. Autrement dit, le pouvoir transite par les individus, il ne s'applique pas à eux. $\gg^{88}$ Les personnes sont aux prises les unes avec les autres dans leurs actes, par l'intermédiaire d'objets techniques : plumes et papier, machines à tout propos, formes graphiques, systèmes de fiches, lieux plus ou moins conformés, enveloppes scellées, motos des fel'd"egery, réseaux téléphoniques ou autres, etc. On n'a plus seulement la bureaucratie avec sa croissance et sa prolifération irrépressible, submergeant même Stalin, son ennemi juré, mais une histoire où des humains se sont installés dans des pratiques de travail qui s'inscrivent dans des objets et des lieux autant que dans des projets personnels. C'est à travers cette circularité que se construit un régime administratif qui tente de s'imposer à tous.

Quant à Stalin, il est un metteur en œuvre insistant de dispositifs administratifs pratiques cardinaux (comme le système de fiches qu'il installe et le tourniquet téléphonique qu'il s'arroge) tout comme un amateur du règlement administratif des questions politiques et l'organisateur de la relation fondamentale entre le parti, la police politique et le gouvernement. Au début des années 1920, il s'entoure à la fois d'assassins de masse professionnels comme Ksenofontov et de remarquables spécialistes de la procédure administrative comme Molotov et Mikojan ${ }^{89}$. C'est sur ce fond que se dessine sa haine active des bureaucrates qui, en tant que tels, sont sujets à négliger les fins politiques de l'art administratif bolchevik : chasser la parole subjectivée, réduire dans l'œuf les insurrections, subjuguer les métiers, détruire les solidarités de tous ordres, se concilier les hiérarchies intermédiaires. Enfin, Stalin gouverne à distance presque sans sortir de ses bureaux, dans une attention précise et suspicieuse à tous les actes administratifs au sujet desquels il organise son information pour mieux surveiller les bureaucrates. La personne de Stalin est extrêmement investie dans les pratiques administratives, à la fois comme instituteur et comme veilleur au quotidien ${ }^{90}$.

Dès lors, on peut dire non seulement que la personne des bureaucrates est présente dans la plus impersonnelle des manifestations administratives, mais qu'en Union soviétique, celles-ci sont, depuis le début des années 1920, indexées à la personne de Stalin - comme elles le sont à leur lieu, à leur moment et à leurs objets matériels, pour emprunter le langage de la pragmatique. Chacun est ainsi lié dans

88. Michel Foucault, "Il faut défendre la société ». Cours au Collège de France. 1976, Paris, Gallimard-Seuil, 1997, p. 26.

89. Sur Ksenofontov, voir Pavel Chinsky, Staline, archives inédites, 1926-1937, Paris, Berg International, 2001, 155 p. Mikojan, « le chef très calé du Narkomtorg » (le commissariat du peuple au Commerce), qui pratiquait habilement dans les années 1920 l'art de transformer les décrets en ordre d'application, principe qu'ignorait Jakovlev, nommé chef de la RKI, l'Inspection ouvrière et paysanne, Moshe Lewin, "Bureaucracy and the Stalinist state », in Ian Kershaw, Moshe Lewin, dirs, Stalinism and nazism, Cambridge, Cambridge University Press, 1997, p. 59.

90. Voir Yves Cohen, « Des lettres comme action : Stalin au début des années 1930 vu depuis le fonds Kaganovič », Cahiers du Monde russe, 38, 3, juil.-sept. 1997, p. 307-346. 
ses propres actes à Stalin. L'histoire de l'administration soviétique, en tant qu'elle est une police généralisée, ne saurait pas plus s'affranchir de ce lien multiforme aux personnes - la personne des opérateurs comme celle de Stalin - que de la conformation matérielle de ses pratiques : c'est tout un.

Centre de recherches historiques

\section{EHESS}

54, boulevard Raspail 75006 Paris

coheny@msh-paris.fr 